\title{
HETEROPHILIOUS DYNAMICS ENHANCES CONSENSUS
}

\author{
SEBASTIEN MOTSCH AND EITAN TADMOR
}

\begin{abstract}
We review a general class of models for self-organized dynamics based on alignment. The dynamics of such systems is governed solely by interactions among individuals or "agents", with the tendency to adjust to their 'environmental averages'. This, in turn, leads to the formation of clusters, e.g., colonies of ants, flocks of birds, parties of people, rendezvous in mobile networks, etc. A natural question which arises in this context is to understand when and how clusters emerge through the self-alignment of agents, and what type of "rules of engagement" influence the formation of such clusters. Of particular interest to us are cases in which the self-organized behavior tends to concentrate into one cluster, reflecting a consensus of opinions, flocking of birds, fish or cells, rendezvous of mobile agents, and in general, concentration of other traits intrinsic to the dynamics.

Many standard models for self-organized dynamics in social, biological and physical science assume that the intensity of alignment increases as agents get closer, reflecting a common tendency to align with those who think or act alike. Moreover, "Similarity breeds connection," reflects our intuition that increasing the intensity of alignment as the difference of positions decreases, is more likely to lead to a consensus. We argue here that the converse is true: when the dynamics is driven by local interactions, it is more likely to approach a consensus when the interactions among agents increase as a function of their difference in position. Heterophily - the tendency to bond more with those who are different rather than with those who are similar, plays a decisive rôle in the process of clustering. We point out that the number of clusters in heterophilious dynamics decreases as the heterophily dependence among agents increases. In particular, sufficiently strong heterophilious interactions enhance consensus.
\end{abstract}

\section{Contents}

1. Introduction

2. Global interactions and unconditional emergence of consensus 6

3. Local interactions and clustering

4. $K=1$ : uniform connectivity implies consensus 18

5. Heterophilious dynamics enhances consensus - simulations

6. Heterophilious dynamics with a fixed-number of neighbors 27

7. Self-alignment dynamics with discrete time steps

8. Mean-field limits: self-organized hydrodynamics

9. Further reading on self-organized dynamics

References

Date: October 15, 2018.

1991 Mathematics Subject Classification. 92D25,74A25,76N10.

Key words and phrases. Agent-based models, self-alignment, heterophilious dynamics, clusters, consensus, flocking, active sets, connectivity of graphs, mean-field limits, kinetic equations, hydrodynamics.

Acknowledgments. S.M. would like to thank the support of the Center for Scientific Computation And Mathematical Modeling (CSCAMM) where this research was performed. We thank the anonymous referee who brought to our attention several references which helped improving an earlier version of the paper. The work is supported by NSF grants DMS10-08397, RNMS11-07444 (KI-Net) and ONR grant N00014-1210318. 


\section{INTRODUCTION}

Nature and human societies offer many examples of self-organized behavior. Ants form colonies to coordinate the construction of a new nest, birds form flocks which fly in the same direction, mobile networks are sought to form a coordinated rendezvous and human crowd form parties to reach a consensus when choosing a leader. The self-organized aspect of such systems is their dynamics, governed solely by interactions among its individuals or "agents", which tend to cluster into colonies, flocks, parties, etc. A natural question which arises in this context is to understand when and how clusters emerge through the self-interactions of agents, and what type of "rules of engagement" influence the formation of such clusters. Of particular interest to us are cases in which the self-organized behavior tends to concentrate into one cluster, reflecting a consensus of opinions, flocking of birds, fish or cells, rendezvous of mobile networks, and in general, concentration around other positions intrinsic to the selforganized dynamics. Generically, we will refer to this process as concentration around an emerging consensus.

Many models have been introduced to appraise the emergence of consensus. Representative examples can be found in [12, 35, 37, 49, 63, 88, 107, 113, and we refer the reader to a more comprehensive list of references surveyed in section 9 below. The starting point for our discussion is a general framework which embed several types of models describing self-organized dynamics. We consider the evolution of $N$ agents, each of which is identified by its "position" $\mathbf{p}_{i}(t) \in \mathbb{R}^{d}$. The position $\mathbf{p}_{i}(t)$ may account for opinion, velocity, or other attributes of agent " $i$ " at time $t$. Each agent adjusts its position according to the position of his neighbors:

$$
\frac{d}{d t} \mathbf{p}_{i}=\alpha \sum_{j \neq i} a_{i j}\left(\mathbf{p}_{j}-\mathbf{p}_{i}\right), \quad a_{i j} \geq 0 .
$$

This provides a rather general description for processes of alignment. Here, $\alpha>0$ is a scaling parameter and the coefficients $a_{i j}$ quantify the strength of influence between agents $i$ and $j$ : the larger $a_{i j}$ is, the more weight is given to agent $j$ to align itself with agent $i$, based on the difference of their positions $\mathbf{p}_{i}-\mathbf{p}_{j}$. The underlying fundamental assumption here is that agents do not react to the position of others but to their differences relative to other agents. In particular, the $a_{i j}$ 's themselves are allowed to depend on the relative differences, $\mathbf{p}_{i}-\mathbf{p}_{j}$. Indeed, we consider nonlinear models (1.1) where

$$
a_{i j}=a_{i j}(\mathcal{P}(t)), \quad \mathcal{P}(t):=\left\{\mathbf{p}_{k}(t)\right\}_{k} .
$$

We emphasize the nonlinear aspect of the alignment models 1.1): the intricate aspect of such models is the nonlinear dependence of the influence matrix on the dynamics, $a_{i j}=a_{i j}(\mathcal{P}(t))$. We ignore two other important processes involved in self-organized dynamics as advocated in the pioneering work of Reynolds, [93], namely, the short-range repulsion (or avoidance) and the long-range cohesion (or attraction), and we refer to recent works driven by the balance of these two processes in e.g., [8, 47, 50, 79, 84, 104. Our purpose here is to shed light on the role of mid-range alignment which covers the important zone "trapped" between the short-range attraction and long-range repulsion.

We distinguish between two main classes of self-alignment models. In the global case, the rules of engagement are such that every agent is influenced by every other agent, $a_{i j}>$ $\eta>0$. The dynamics in this case is driven by global interactions. We have a fairly good understanding of the large time dynamics of such models; an incomplete list of recent works in this direction includes [11, 18, 37, 44, 60, 61, 63, 69, 75, 88], and the references therein. Global interactions which are sufficiently strong lead to unconditional consensus in the sense that all 
initial configurations of agents concentrate around an emerging limit state, the "consensus" $\mathbf{p}^{\infty}$

$$
\mathbf{p}_{i}(t) \stackrel{t \rightarrow \infty}{\longrightarrow} \mathbf{p}^{\infty}
$$

The first part of the paper, section 2, contains an overview of the concentration dynamics in such global models, from the perspective of the general framework of (1.1).

In more realistic models, however, interactions between agents are limited to their local neighbors, [1, 4, 36, 71, 93. The behavior of local models where some of the $a_{i j}$ may vanish, requires a more intricate analysis. In the general scenario for such local models, discussed in section 3, agents tend to concentrate into one or more separate clusters. The particular case in which agents concentrate into one cluster, that is the emergence of a consensus or a flock, depends on the propagation of uniform connectivity of the underling (weighted) graph associated with the adjacency matrix, $\left\{a_{i j}\right\}$. This issue is explored in section 4 where we show that connectivity implies consensus. Thus, the question of consensus for local models is turned into the question of persistence of connectivity over time. Note that even if the initial configuration is assumed connected, then there is still a possibility of losing connectivity as the $a_{i j}$ 's may vary in time together with the positions $\mathcal{P}(t)$. The open question of tracing the propagation of connectivity in time for general class of local models 1.1 plays an important role in many applications, beyond the implication of emerging consensus. As an example we mention engineering applications to sensor-based networks, from automatic traffic control and wireless communication to production systems and mobile robot networks, e.g., [64, 71, 89, 90, 94, 112, 113 , and the references therein.

Many standard models for self-organized dynamics in social, biological and physical science assume that the dependence of $a_{i j}$ decreases as a function of $\left|\mathbf{p}_{i}-\mathbf{p}_{j}\right|$, where $|\cdot|$ is a problemdependent proper metric to measure a difference of positions, opinions, etc. The statement that "Birds of feature flock together" reflects a common tendency to align with those who think or act alike, 69, 77, 83]. Moreover, "Similarity breeds connection," reflects the intuitive scenarios in which the influence coefficients $a_{i j}$ increase as the difference of positions $\left|\mathbf{p}_{i}-\mathbf{p}_{j}\right|$ decreases: the more the $a_{i j}$ 's increase, the more likely it is to lead to a consensus. But in fact, we argue here that the converse is true: for a self-organized dynamics driven by local interactions, it is more likely to approach a consensus when the interaction among agents increases as a function of their difference $\left|\mathbf{p}_{i}-\mathbf{p}_{j}\right|$. Heterophily - the tendency to bond more with the different rather than with those who are similar, plays a decisive rôle in the clustering of (1.1). The consensus in heterophilious dynamics is explored in the second part of the paper, in terms of local interactions of the form, $a_{i j}=\phi\left(\left|\mathbf{p}_{i}-\mathbf{p}_{j}\right|\right)$, where $\phi(\cdot)$ is a compactly supported influence function which is increasing over its support. In section 5 we report our extensive numerical simulations which confirm the counter-intuitive phenomenon, where the number of clusters decreases as the heterophilious dependence increases; in particular, if $\phi$ is increasing fast enough then the corresponding dynamics concentrate into one cluster, that is, heterophilious dynamics enhances consensus. We mention in passing the scenario of "extreme heterophily" advocated in [71, 112, 113], where distributed coordination is governed by local influence function $\phi(\cdot)$ which grows to infinity as it approaches the right edge of its support, in order to create an energy barrier which enforces connectivity and hence consensus. We are not unaware that this phenomenon of enhanced consensus in the presence of heterophilious interactions, may have intriguing consequences in different areas other than social networks, e.g., global bonding in atomic scales, avoiding materials' fractures in mesoscopic scales, or "cloud" formations in macroscopic scales. 
In the rest of the paper, we address a few important extensions of the self-alignment models outlined above. These extensions are still work in progress and we by no means try to be comprehensive. In section 6 we turn our attention to nearest neighbor dynamics. Careful 3D observations made by the StarFlag project, [25, 26, 27], showed that interactions of birds are driven by topological neighborhoods, involving a fixed number of nearby birds, instead of geometric neighborhoods involving a fixed radius of interaction. Here we prove that in the simplest case of two nearest neighbor dynamics, connectivity propagates in time and consensus follows for influence functions which are non-decreasing on their compact support. In section 7 we turn our attention to fully discrete models for self-alignment. The large time evolution in discrete time-steps, e.g., the opinion of dynamics in [11, 12, 75], may depend on the time-step $\Delta t$. Here, we show that the semi-discrete framework for global and local self-alignment outlined in sections 25 can be extended, mutatis mutandis, to the fully-discrete case. In particular, we recover a decreasing Lyapunov functional, a fully-discrete analogue of the semi-discrete clustering analysis in section 4.2 . Finally, in section 8 we discuss the passage from the agentbased description to mean-field limits as the number of agents, or "particles" tends to be large enough. There is a growing literature on kinetic descriptions of such models, [21, 22, 23, 49, 51, 61 and the references therein. Here we focus our attention on the hydrodynamic descriptions of self-organized opinion dynamics and flocking. The closing section 9 is devoted to a more detailed discussion on the broader subject of self-organized dynamics. Since a comprehensive review of this multidisciplinary subject is beyond the scope of this paper, in particular, we include a selection of references, classified into several complementary categories of different disciplines, models, scales, approaches and patterns.

1.1. Examples of opinion dynamics and flocking. Models for self-organized dynamics (1.1) have appeared in a large variety of different contexts, including load balancing in computer networks, evolution of languages, gossiping, algorithms for sensor networks, emergence of flocks, herds, schools and other biological "clustering", pedestrian dynamics, ecological models, peridynamic elasticity, multi-agent robots, models for opinion dynamics, economic networks and more; a detailed list of references is surveyed in section 9 below.

To demonstrate the general framework for self-alignment dynamics (1.1), we shall work with two main concrete examples. The first models opinions dynamics. In these models, $N$ agents, each with vector of opinions quantified by $\mathbf{p}_{i} \rightsquigarrow \mathbf{x}_{i} \in \mathbb{R}^{d}$, interact with each other according to the first-order system,

$$
\frac{d}{d t} \mathbf{x}_{i}=\alpha \sum_{j \neq i} a_{i j}\left(\mathbf{x}_{j}-\mathbf{x}_{i}\right) \quad, \quad a_{i j}=\frac{\phi\left(\left|\mathbf{x}_{j}-\mathbf{x}_{i}\right|\right)}{N} .
$$

Here, $0<\phi<1$ is the scaled influence function which acts on the "difference of opinions", $\left|\mathbf{x}_{i}-\mathbf{x}_{j}\right|$. The metric $|\cdot|$ needs to be properly interpreted, adapted to the specific context of the problem at hand. Another model for interaction of opinions is

$$
\frac{d}{d t} \mathbf{x}_{i}=\alpha \sum_{j \neq i} a_{i j}\left(\mathbf{x}_{j}-\mathbf{x}_{i}\right) \quad, \quad a_{i j}=\frac{\phi_{i j}}{\sum_{k} \phi_{i k}}, \quad \phi_{i j}:=\phi\left(\left|\mathbf{x}_{j}-\mathbf{x}_{i}\right|\right) .
$$

The classical Krause model for opinion dynamics [75, 12] is a time-discretization of (1.2b), which will be discussed in section 7 below. Observe that the adjacency matrix $\left\{a_{i j}\right\}$ in the first model (1.2a) is symmetric while in the second model, $1.2 \mathrm{~b})$, it is not.

Another branch of models have been proposed to describe flocking. These are second-order models where the observed property is the velocity of birds, $\mathbf{p}_{i} \mapsto \mathbf{v}_{i} \in \mathbb{R}^{d}$, which are coupled 
to their location $\mathbf{x}_{i} \in \mathbb{R}^{d}$. The flocking model of Cucker and Smale (C-S) has received a considerable attention in recent years, [37, 38, 61, 18, 60],

$$
\frac{d}{d t} \mathbf{v}_{i}=\alpha \sum_{j \neq i} a_{i j}\left(\mathbf{v}_{j}-\mathbf{v}_{i}\right) \quad, \quad a_{i j}=\frac{\phi\left(\left|\mathbf{x}_{j}-\mathbf{x}_{i}\right|\right)}{N} \quad \text { where } \quad \frac{d}{d t} \mathbf{x}_{i}=\mathbf{v}_{i} .
$$

In C-S model, alignment is carried out by isotropic averaging. In [88] we advocated a more realistic alignment-based model for flocking, where alignment is based on the relative influence, similar to (1.2b),

$$
\frac{d}{d t} \mathbf{v}_{i}=\alpha \sum_{j \neq i} a_{i j}\left(\mathbf{v}_{j}-\mathbf{v}_{i}\right) \quad, \quad a_{i j}=\frac{\phi_{i j}}{\sum_{k} \phi_{i k}} \quad \text { with } \quad \phi_{i j}:=\phi\left(\left|\mathbf{x}_{j}-\mathbf{x}_{i}\right|\right) .
$$

Again, C-S model is based on a symmetric adjacency matrix, $\left\{a_{i j}\right\}$, while symmetry is lost in (1.3b), i.e. $a_{i j} \neq a_{j i}$.
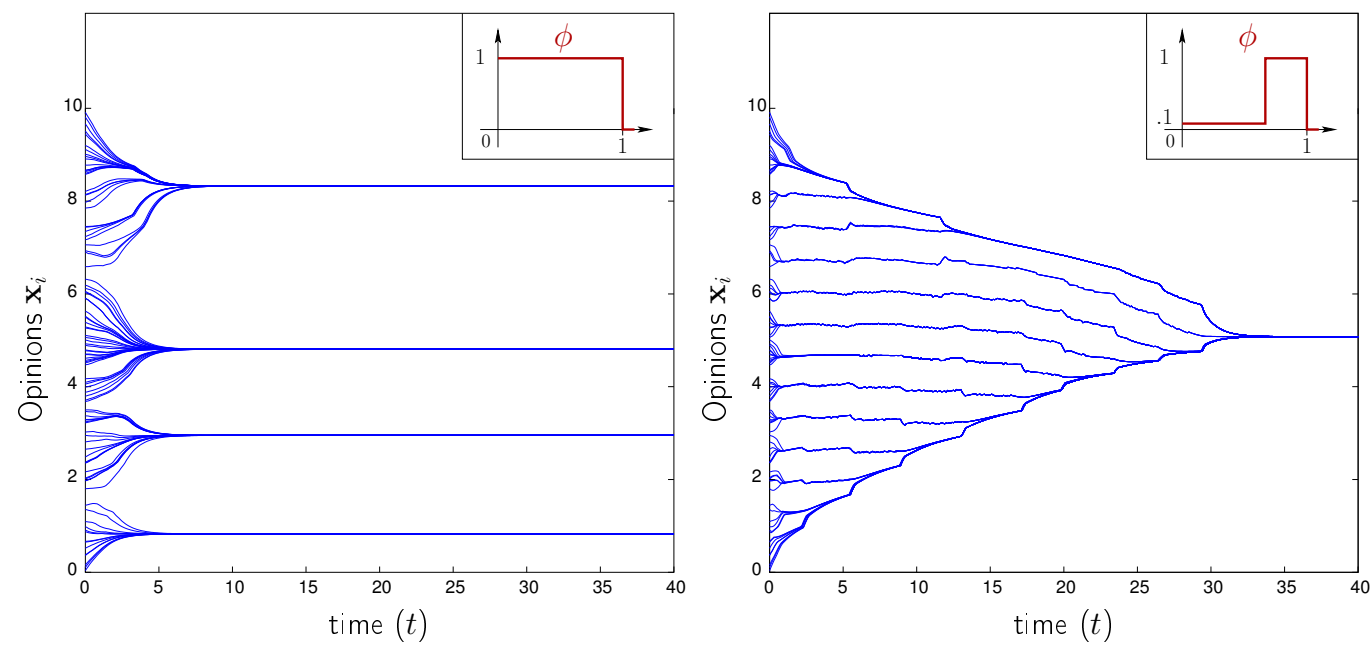

FiguRE 1.1. Evolution in time of the consensus model for two different influence functions $\phi$ (Left figure: $\phi(r)=\chi_{[0,1]}$, Right figure: $\left.\phi(r)=.1 \chi_{[0,1 / \sqrt{2}]}+\chi_{[1 / \sqrt{2}, 1]}\right)$. By diminishing the influence of close neighbors (Right figure), we enhance the emergence of a consensus. Simulations are started with the same initial condition (100 agents uniformly distributed on $[0,10])$.

The models for opinion and flocking dynamics 1.2 , and respectively, 1.3 , can be written in the unified form

$$
\frac{d}{d t} \mathbf{p}_{i}=\alpha \sum_{j=1}^{N} a_{i j}\left(\mathbf{p}_{j}-\mathbf{p}_{i}\right), \quad a_{i j}=\frac{1}{\sigma_{i}} \phi\left(\left|\mathbf{x}_{i}-\mathbf{x}_{j}\right|\right) .
$$

In the opinion dynamics, $\mathbf{p} \mapsto \mathbf{x}$; in the flocking dynamics, $\mathbf{p} \mapsto \dot{\mathbf{x}}$. The degree $\sigma_{i}=N$ in the symmetric models, or $\sigma_{i}=\sum_{j \neq i} \phi\left(\left|\mathbf{x}_{i}-\mathbf{x}_{j}\right|\right)$ in the non-symmetric models. The local vs. global behavior of these models hinges on the behavior of the influence function, $\phi$. If the support of $\phi$ is large enough to cover the convex hull of $\mathcal{P}(0)=\left\{\mathbf{p}_{k}(0)\right\}_{k}$, then global interactions will yield unconditional consensus or flocking. On the other hand, if $\phi$ is locally supported, then the group dynamics in (1.4) depends on the connectivity of the underlying graph, $\left\{a_{i j}\right\}$. In particular, if the overall connectivity is lost over time, then each connected component may 
lead to a separate cluster. Heterophilious self-organized dynamics is characterized by a locally supported influence function, $\phi$, which is increasing as a function of the mutual differences, $\phi_{i j}=\phi\left(\left|\mathbf{x}_{i}-\mathbf{x}_{j}\right|\right)$. The more heterophilious the dynamics is, in the sense that its influence function has a steeper increase over its compact support, the more it tends to concentrate in the sense of approaching a smaller number of clusters. In particular, heterophilious dynamics is more likely to lead to a consensus as demonstrated for example, in figure 1.1 (and is further documented in figures 5.2 and 5.7 below). Observe that the only difference between the two models depicted in figure 1.1 is that the influence in the immediate neighborhood (of radius

$r \leq 1 / \sqrt{2}$ ) was decreased, from $\phi=1 \chi_{[0,1]}$ (on the left) into $\phi=0.1 \chi_{[0,1 / \sqrt{2}]}+\chi_{[1 / \sqrt{2}, 1]}$ (on the right): this was sufficient to enhance the four-party clustering on the left to turn into a consensus shown on the right.

\section{Global interactions and unconditional emergence of Consensus}

In this section we derive explicit conditions for global self-organized dynamics (1.1) to concentrate around an emerging consensus. Our starting point is a convexity argument which is valid for any adjacency matrix $A=\left\{a_{i j}\right\}$, whether symmetric or not. We begin by noting without loss of generality, that $A$ may be assumed to be row-stochastic,

$$
\sum_{j} a_{i j}=1, \quad i=1, \ldots, N
$$

Indeed, by rescaling $\alpha$ if necessary we have $\sum_{j \neq i} a_{i j} \leq 1$, and 2.1 holds when we set $a_{i i}:=$ $1-\sum_{j \neq i} a_{i j} \geq 0$. We rewrite 1.1 in the form

$$
\frac{d}{d t} \mathbf{p}_{i}=\alpha\left(\overline{\mathbf{p}}_{i}-\mathbf{p}_{i}\right), \quad \overline{\mathbf{p}}_{i}:=\sum_{j=1}^{N} a_{i j} \mathbf{p}_{j} .
$$

Thus, if we let $\Omega(t)$ denote the convex hull of the properties $\left\{\mathbf{p}_{k}\right\}_{k}$, then according to $(2.2), \mathbf{p}_{i}$ is relaxing to the average value $\overline{\mathbf{p}}_{i} \in \Omega(t)$, while the boundary of $\Omega$ is a barrier for the dynamics. It follows that the positions in the general self-organized model (1.1) remain bounded.

Proposition 2.1. The convex hull of $\mathbf{p}(t)$ is decreasing in time in the sense that the convex hull, $\Omega(t):=\operatorname{Conv}\left(\left\{\mathbf{p}_{i}(t)\right\}_{i \in[1, N]}\right)$, satisfies

$$
\Omega\left(t_{2}\right) \subset \Omega\left(t_{1}\right), \quad t_{2}>t_{1} \geq 0 .
$$

Moreover, we have

$$
\max _{i}\left|\mathbf{p}_{i}(t)\right| \leq \max _{i}\left|\mathbf{p}_{i}(0)\right| .
$$

Proof. We verify (2.4) for a general vector norm $|\cdot|$ which we characterize in terms of its dual $|\mathbf{w}|_{*}=\sup _{\mathbf{w} \neq 0}\langle\mathbf{w}, \mathbf{z}\rangle /|\mathbf{z}|$ so that $|\mathbf{p}|=\sup \langle\mathbf{p}, \mathbf{w}\rangle /|\mathbf{w}|_{*}$. Let $\mathbf{w}=\mathbf{w}(t)$ denotes the maximal dual vector of $\mathbf{p}_{i}(t)$, so that $\left\langle\mathbf{p}_{i}, \mathbf{w}\right\rangle=\left|\mathbf{p}_{i}\right|$, then

$$
\left\langle\dot{\mathbf{p}}_{i}, \mathbf{w}\right\rangle=\alpha\left(\left\langle\overline{\mathbf{p}}_{i}, \mathbf{w}\right\rangle-\left\langle\mathbf{p}_{i}, \mathbf{w}\right\rangle\right) \leq \alpha\left(\left|\overline{\mathbf{p}}_{i}\right|-\left|\mathbf{p}_{i}\right|\right) .
$$

Since $\left\langle\mathbf{p}_{i}, \dot{\mathbf{w}}\right\rangle \leq 0$ we have

$$
\frac{d}{d t}\left|\mathbf{p}_{i}(t)\right|=\left\langle\dot{\mathbf{p}}_{i}, \mathbf{w}\right\rangle+\left\langle\mathbf{p}_{i}, \dot{\mathbf{w}}\right\rangle \leq \alpha\left(\left|\overline{\mathbf{p}}_{i}(t)\right|-\left|\mathbf{p}_{i}(t)\right|\right),
$$

and finally, $\left|\overline{\mathbf{p}}_{i}(t)\right| \leq \max _{i}\left|\mathbf{p}_{i}(t)\right|$ yields $(2.4)$. 


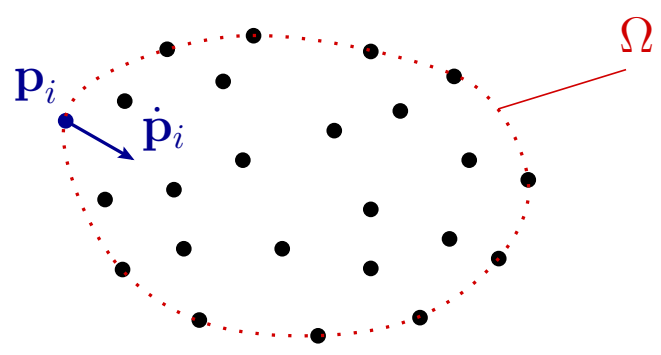

Figure 2.1. The convex hull $\Omega$ of the positions $\mathbf{p}_{i}$.

Remark. Since the models of opinion dynamics and flocking dynamics (1.4) are translation invariant in the sense of admitting the family of solutions $\left\{\mathbf{p}_{i}-\mathbf{c}\right\}$, then for any fixed state $\mathbf{c}$, proposition 2.1 implies

$$
\max _{i}\left|\mathbf{p}_{i}(t)-\mathbf{c}\right| \leq \max _{i}\left|\mathbf{p}_{i}(0)-\mathbf{c}\right| .
$$

Consensus and flocking are achieved when the decreasing $\Omega(t)$ shrinks to a limit point $\Omega(t) \stackrel{t \rightarrow \infty}{\longrightarrow}\left\{\mathbf{p}^{\infty}\right\}$

$$
\max _{i}\left|\mathbf{p}_{i}(t)-\mathbf{p}^{\infty}\right| \stackrel{t \rightarrow \infty}{\longrightarrow} 0 .
$$

There are various approaches, not unrelated, to derive conditions which ensure unconditional consensus or flocking. We shall mention two: an $L^{\infty}$ contraction argument and an $L^{2}$ energy method based on spectral analysis.

2.1. An $L^{\infty}$ approach: contraction of diameters. Proposition 2.1 tells us that $\left\{\mathbf{p}_{i}(t)\right\}_{i}$ remain uniformly bounded and the diameter, $\max _{i j}\left|\mathbf{p}_{i}(t)-\mathbf{p}_{j}(t)\right|$, is non-increasing in time. In order to have concentration, however, we need to verify that the diameter of $\mathbf{p}(t)$ decays to zero. The next proposition quantifies this decay rate.

Theorem 2.2. Consider the self-organized model (1.1) with a raw stochastic adjacency matrix A, 2.1). Let

$$
[\mathbf{p}]:=\max _{i j}\left|\mathbf{p}_{i}-\mathbf{p}_{j}\right|
$$

denote the diameter of the position vector $\mathbf{p}$. Then the diameter satisfies the concentration estimate

$$
\frac{d}{d t}[\mathbf{p}(t)] \leq-\alpha \eta_{A(\mathcal{P}(t))}[\mathbf{p}(t)], \quad \eta_{A}:=\min _{i j} \sum_{k} \min \left\{a_{i k}, a_{j k}\right\} .
$$

In particular, if there is a slow decay of the concentration factor so that $\int_{\eta_{A(\mathcal{P}(s))}}^{\infty} d s=\infty$, then the agents concentrate in the sense that

$$
\Theta(t):=\int^{t} \eta_{A(\mathcal{P}(s))} d s \stackrel{t \rightarrow \infty}{\longrightarrow} \infty \quad \rightsquigarrow \quad \lim _{t \rightarrow \infty} \max _{i, j}\left|\mathbf{p}_{i}(t)-\mathbf{p}_{j}(t)\right|=0 .
$$

Moreover, if the decay of the concentration factor is slow enough in the sense that $\int^{\infty} \exp (-\alpha \Theta(s)) d s<$ $\infty$, then there is an emerging consensus $\mathbf{p}^{\infty} \in \Omega(0)$,

$$
\int^{\infty} e^{-\alpha \Theta(t)} d t<\infty \quad \rightsquigarrow \quad\left|\mathbf{p}_{i}(t)-\mathbf{p}^{\infty}\right| \lesssim e^{-\alpha \Theta(t)}[\mathbf{p}(0)] \quad \text { for all } i=1, \ldots, N .
$$

Remark. We note that theorem 2.2 applies to any vector norm $|\cdot|$. 
Proof. We begin with the following estimate which quantifies the contractivity of the row stochastic $A$ in the induced vector semi-norm [·] (since this bound is solely due to the convexity of the row stochastic $A$, we suppress the time-dependence of $\mathbf{p}$ and $\overline{\mathbf{p}}=A \mathbf{p}$ ),

$$
[A \mathbf{p}] \leq\left(1-\eta_{A}\right)[\mathbf{p}], \quad[\mathbf{p}]=\max _{i j}\left|\mathbf{p}_{i}-\mathbf{p}_{j}\right|, \quad 1-\eta_{A}=\frac{1}{2} \sum_{k}\left|a_{i k}-a_{j k}\right| .
$$

The estimate 2.7 in its $\ell^{1}$-dual form for column stochastic matrices goes back to Dobrushin [46], and his so-called coefficient of ergodicity, $\eta_{A}$, was later used to quantify the relative entropy in discrete Markov processes [30, 31] and the contractivity in models of opinion dynamics [75]. For completeness, we proceed with the proof for general vector norms $|\cdot|$. Fix any $i$ and $j$ which are to be chosen later, and set $\eta_{k}:=\min \left\{a_{i k}, a_{j k}\right\}$ so that $a_{i k}-\eta_{k}$ and $a_{j k}-\eta_{k}$ are non-negative. Then, for arbitrary $\mathbf{w} \in \mathbb{R}^{d}$ we have,

$$
\begin{aligned}
\left\langle\overline{\mathbf{p}}_{i}-\overline{\mathbf{p}}_{j}, \mathbf{w}\right\rangle & =\sum_{k} a_{i k}\left\langle\mathbf{p}_{k}, \mathbf{w}\right\rangle-\sum_{k} a_{j k}\left\langle\mathbf{p}_{k}, \mathbf{w}\right\rangle \\
& =\sum_{k}\left(a_{i k}-\eta_{k}\right)\left\langle\mathbf{p}_{k}, \mathbf{w}\right\rangle-\sum_{k}\left(a_{j k}-\eta_{k}\right)\left\langle\mathbf{p}_{k}, \mathbf{w}\right\rangle \\
& \leq \sum_{k}\left(a_{i k}-\eta_{k}\right) \max _{k}\left\langle\mathbf{p}_{k}, \mathbf{w}\right\rangle-\sum_{k}\left(a_{j k}-\eta_{k}\right) \min _{k}\left\langle\mathbf{p}_{k}, \mathbf{w}\right\rangle \\
& =\left(1-\eta_{A}\right)\left(\max _{k}\left\langle\mathbf{p}_{k}, \mathbf{w}\right\rangle-\min _{k}\left\langle\mathbf{p}_{k}, \mathbf{w}\right\rangle\right) \\
& \leq\left(1-\eta_{A}\right) \max _{k \ell}\left\langle\mathbf{p}_{k}-\mathbf{p}_{\ell}, \mathbf{w}\right\rangle \leq\left(1-\eta_{A}\right) \max _{k, \ell}\left|\mathbf{p}_{k}-\mathbf{p}_{\ell} \| \mathbf{w}\right|_{*}
\end{aligned}
$$

In the last step, we characterize the norm $|\cdot|$ by its dual $|\mathbf{w}|_{*}=\sup _{\mathbf{w} \neq 0}\langle\mathbf{w}, \mathbf{z}\rangle /|\mathbf{z}|$ so that $\langle\mathbf{z}, \mathbf{w}\rangle \leq|\mathbf{z}||\mathbf{w}|_{*}$. Now, choose $i$ and $j$ as a maximal pair such that $[\overline{\mathbf{p}}]=\left|\overline{\mathbf{p}}_{i}-\overline{\mathbf{p}}_{j}\right|$; we then have

$$
[A \mathbf{p}] \equiv[\overline{\mathbf{p}}]=\left|\overline{\mathbf{p}}_{i}-\overline{\mathbf{p}}_{j}\right|=\sup _{\mathbf{w} \neq 0} \frac{\left\langle\overline{\mathbf{p}}_{i}-\overline{\mathbf{p}}_{j}, \mathbf{w}\right\rangle}{|\mathbf{w}|_{*}} \leq\left(1-\eta_{A}\right) \max _{k, \ell}\left|\mathbf{p}_{k}-\mathbf{p}_{\ell}\right|
$$

and 2.7) now follows.

Next, we consider the discrete time-marching system associated with (1.1),

$$
\frac{\mathbf{p}(t+\Delta t)-\mathbf{p}(t)}{\Delta t}=\alpha(A \mathbf{p}(t)-\mathbf{p}(t)) \text {. }
$$

Using (2.7) we obtain

$$
[\mathbf{p}(t+\Delta t)]=[(1-\alpha \Delta t) \mathbf{p}(t)+\alpha \Delta t A \mathbf{p}(t)] \leq(1-\alpha \Delta t)[\mathbf{p}(t)]+\alpha \Delta t\left(1-\eta_{A}\right)[\mathbf{p}(t)],
$$

or after rearrangement,

$$
\frac{[\mathbf{p}(t+\Delta t)]-[\mathbf{p}(t)]}{\Delta t} \leq-\alpha \eta_{A}[\mathbf{p}(t)]
$$

and the desired bound 2.5 follows by letting $\Delta t \rightarrow 0$. In particular, we have

$$
\max _{i j}\left|\mathbf{p}_{i}(t)-\mathbf{p}_{j}(t)\right| \leq \exp \left(-\alpha \int_{0}^{t} \eta_{A(\mathcal{P}(s))} d s\right)[\mathbf{p}(0)] \stackrel{t \rightarrow \infty}{\longrightarrow} 0
$$


which proves (2.6a). Moreover,

$$
\begin{aligned}
\left|\mathbf{p}_{i}\left(t_{2}\right)-\mathbf{p}_{i}\left(t_{1}\right)\right| & =\left|\int_{\tau=t_{1}}^{t_{2}} \dot{\mathbf{p}}_{i}(\tau) d \tau\right| \leq \alpha \max _{i j} \int_{\tau=t_{1}}^{t_{2}}\left|\mathbf{p}_{i}(\tau)-\mathbf{p}_{j}(\tau)\right| d s \\
& \leq \alpha \int_{\tau=t_{1}}^{t_{2}} \exp (-\alpha \Theta(\tau)) d \tau[\mathbf{p}(0)], \quad \Theta(\tau)=\int_{0}^{\tau} \eta_{A(\mathcal{P}(s)} d s,
\end{aligned}
$$

which tends to zero, $\left|\mathbf{p}_{i}\left(t_{2}\right)-\mathbf{p}_{i}\left(t_{1}\right)\right| \rightarrow 0$ for $t_{2}>t_{1} \gg 1$, thanks to our assumption (2.6b). It follows that the limit $\mathbf{p}_{i}(t) \stackrel{t \rightarrow \infty}{\longrightarrow} \mathbf{p}_{i}^{\infty}$ exists, and hence all agents concentrate around the same limit position, an emerging consensus $\mathbf{p}^{\infty} \in \Omega(0)$. The concentration rate estimate (2.6b) follows from (2.8).

Theorem 2.2 relates the emergence of consensus or flocking of $\dot{\mathbf{p}}=A \mathbf{p}-\mathbf{p}$ to the behavior of $\int^{t} \eta_{A(\mathcal{P}(s))} d s \uparrow \infty$, and to this end we seek lower-bounds on the "concentration factor" $\eta_{A}$, which are easily checkable in terms of the entries of $A$. This brings us to the following definition.

Definition (Active sets [88]). Fix $\theta>0$. The active set, $\Lambda(\theta)$, is the set of agents which influence every other agent "more" than $\theta$,

$$
\Lambda(\theta):=\left\{j \mid a_{i j} \geq \theta \text { for any } i\right\} .
$$

Observe that since $a_{i j}$ changes in time, $a_{i j}=a_{i j}(\mathcal{P}(t))$, the number of agents in the active set $\Lambda(\theta)$ is a time dependent quantity, denoted $\lambda(\theta)=\lambda(\theta, t):=|\Lambda(\theta, t)|$.

The straightforward lower bound, $\eta_{A} \geq \max _{\theta} \theta \cdot \lambda(\theta)$ yields

Corollary 2.3. The diameter of the self-organized model (1.1) with a stochastic adjacency matrix A, (2.1) satisfies the concentration estimate

$$
\frac{d}{d t}[\mathbf{p}(t)] \leq-\alpha\left(\max _{\theta} \theta \cdot \lambda(\theta, t)\right)[\mathbf{p}(t)] .
$$

In particular, the lower bound $\eta_{A} \geq N \min _{i j} a_{i j}$, corresponding to $\theta=\min _{i j} a_{i j}$ with $\lambda(\theta, t)=$ $N$, yields [61]

$$
\left|\mathbf{p}(t)-\mathbf{p}^{\infty}\right| \lesssim \exp \left(-\alpha N \int_{0}^{t} m(s) d s\right)[\mathbf{p}(0)], \quad m(s):=\min _{i j} a_{i j}(s) .
$$

Remark. The bound (2.10) is an improvement of the "flocking" estimate [88, Lemma 3.1]

$$
\frac{d}{d t}[\mathbf{p}(t)] \leq-\alpha\left(\max _{\theta} \theta \cdot \lambda(\theta, t)\right)^{2}[\mathbf{p}(t)]
$$

Corollary 2.3 is a useful tool to verify consensus and flocking behavior for general adjacency matrices $A=\left\{a_{i j}\right\}$, whether symmetric or not. We demonstrate its application with the following sufficient condition for the emergence of a consensus in the opinion models (1.2). In either the symmetric or non-symmetric case,

$$
a_{i j}=\left\{\begin{array}{c}
\frac{\phi_{i j}}{N} \\
\frac{\phi_{i j}}{\sigma_{i}}
\end{array}\right\} \geq \frac{\phi([\mathbf{x}(t)])}{N}, \quad \sigma_{i}=\sum_{k} \phi_{i k} \leq N .
$$

By proposition 2.1, the diameter $[\mathbf{x}(t)]$ is non-increasing, yielding the lower bound

$$
N a_{i j}(\mathcal{P}(t))=\frac{N}{\sigma_{i}} \phi\left(\left|\mathbf{x}_{i}(t)-\mathbf{x}_{j}(t)\right|\right) \geq \min _{r \leq[\mathbf{x}(t)]} \phi(r) \geq \min _{r \leq[\mathbf{x}(0)]} \phi(r),
$$


which in turns implies the following exponentially fast convergence towards a consensus $\mathbf{x}^{\infty}$.

Proposition 2.4 (Unconditional consensus). Consider the models for opinion dynamics (1.2) with an influence function $\phi(r) \leq 1$, and assume that

$$
m:=\min _{r \leq[\mathbf{x}(0)]} \phi(r)>0 .
$$

Then, there is an exponentially fast convergence towards an emerging consensus $\mathbf{x}^{\infty}$,

$$
\left|\mathbf{x}_{i}(t)-\mathbf{x}^{\infty}\right| \lesssim e^{-\alpha m t}[\mathbf{x}(0)]
$$

Similar arguments apply for the flocking models $(1.3)$ : since $[\mathbf{v}(t)]$ is non-increasing then $[\mathbf{x}(t)] \leq[\mathbf{x}(0)]+t[\mathbf{v}(0)]$ and hence

$$
N a_{i j}(\mathcal{P}(t))=\frac{N}{\sigma_{i}} \phi\left(\left|\mathbf{x}_{i}(t)-\mathbf{x}_{j}(t)\right|\right) \geq \min _{r \leq[\mathbf{x}(t)]} \phi(r) \geq \min _{r \leq[\mathbf{x}(0)]+t[\mathbf{v}(0)]} \phi(r) ;
$$

if $\phi(\cdot)$ is decreasing then we can set $m(t)=\phi([\mathbf{x}(0)]+t[\mathbf{v}(0)])$ and unconditional flocking follows from for corollary 2.3 for sufficiently strong interaction so that $\int^{\infty} \phi(s) d s=\infty$. In fact, a more precise statement of flocking is summarized in the following.

Proposition 2.5 (Unconditional flocking). Consider the flocking dynamics (1.3) with a decreasing influence function $\phi(r) \leq \phi(0) \leq 1$, and assume that

$$
\int^{\infty} \phi(s) d s=\infty
$$

Then, the diameter of positions remains uniformly bounded, $[\mathbf{x}(t)] \leq D_{\infty}<\infty$, and there is an exponentially fast concentration of velocities around a flocking state $\mathbf{v}^{\infty}$,

$$
\left|\mathbf{v}_{i}(t)-\mathbf{v}^{\infty}\right| \lesssim e^{-\alpha m t}[\mathbf{v}(0)], \quad m=\phi\left(D_{\infty}\right) .
$$

Proof. Unlike the first-order models for consensus, the diameter in second-order flocking models, $[\mathbf{x}(t)]$, may increase in time. The bound $D_{\infty}$ stated in 2.15 places a uniform bound on the maximal active diameter. To derive such a bound observe that in the second order flocking models, the evolution of the diameter of velocities satisfies,

$$
\frac{d}{d t}[\mathbf{v}(t)] \leq-\alpha \phi([\mathbf{x}(t)])[\mathbf{v}(t)]
$$

and is coupled with the evolution of positions $[\mathbf{x}(t)]$ : since $\dot{\mathbf{x}}=\mathbf{v}$, we have

$$
\frac{d}{d t}[\mathbf{x}(t)] \leq[\mathbf{v}(t)]
$$

The last two inequalities imply that the following energy functional introduced by Ha and Liu [60],

is decreasing in time,

$$
\mathcal{E}(t):=[\mathbf{v}(t)]+\alpha \int_{0}^{[\mathbf{x}(t)]} \phi(s) d s,
$$

$$
\alpha \int_{[\mathbf{x}(0)]}^{[\mathbf{x}(t)]} \phi(s) d s \leq[\mathbf{v}(0)]-[\mathbf{v}(t)] \leq[\mathbf{v}(0)] .
$$

This, together with our assumption (2.14) yield the existence of a finite $D_{\infty}>[\mathbf{x}(0)]$ such that

$$
\alpha \int_{[\mathbf{x}(0)]}^{[\mathbf{x}(t)]} \phi(s) d s \leq[\mathbf{v}(0)] \leq \alpha \int_{[\mathbf{x}(0)]}^{D_{\infty}} \phi(s) d s .
$$


Thus, the active diameter of positions does not exceed $[\mathbf{x}(t)] \leq D_{\infty}$, and since $\phi$ is assumed decreasing, the minimal interaction is $N a_{i j} \geq \phi([\mathbf{x}(t)]) \geq \phi\left(D_{\infty}\right)$ which yields

$$
\frac{d}{d t}[\mathbf{v}(t)] \leq-\alpha \phi\left(D_{\infty}\right)[\mathbf{v}(t)] .
$$

This concludes the proof of 2.15).

Remark (Global interactions). Proposition 2.4 derives an unconditional consensus under the assumption of global interaction, namely, according to (2.12) every agent interacts with every other agent as

$$
a_{i j} \geq \frac{1}{N} \phi\left(\left|\mathbf{x}_{i}-\mathbf{x}_{j}\right|\right) \geq \frac{m}{N}>0 .
$$

Similarly, the unconditional flocking stated in proposition 2.5 requires global interactions, in the sense of having an influence function (2.14) which is supported over the entire flock. Indeed, if the influence function $\phi$ is compactly supported, supp $\{\phi\}=[0, R]$, then assumption (2.14) tells us that

$$
[\mathbf{v}(0)] \leq \alpha \int_{[\mathbf{x}(0)]}^{R} \phi(s) d s
$$

but according to 2.16$), \alpha \int_{[\mathbf{x}(0)]}^{[\mathbf{x}(t)]} \phi(s) d s \leq[\mathbf{v}(0)]$ and hence the support of $\phi$ remains larger than the diameter of positions, $R \geq[\mathbf{x}(t)]$.

Proposition 2.5 recovers the unconditional flocking results for the C-S model, $\phi(r) \propto(1+$ $r)^{-2 \beta}, \beta>1 / 2$, obtained earlier using spectral analysis, $\ell_{1^{-}}, \ell_{2^{-}}$and $\ell_{\infty^{-b a s e d}}$ estimates [37, 61, 18, 60, 22. The derivations are different, yet they all required the symmetry of the C-S influence matrix, $a_{i j}=\phi_{i j} / N$. Here, we unify and generalize the results, covering both the symmetric and non-symmetric scenarios. In particular, we improve here the unconditional flocking result in the non-symmetric model obtained in [88, theorem 4.1]. Although the tools are different - notably, lack of conservation of momentum $\frac{1}{N} \sum_{i} \mathbf{v}_{i}(t)$ in the non-symmetric case, we nevertheless end up with same condition (2.14) for unconditional flocking.

2.2. Spectral analysis of symmetric models. A more precise description of the concentration phenomena is available for models governed by symmetric influence matrices, $a_{i j}=a_{j i}$, such as (1.2a) and (1.3a). Set $\mathbf{q}_{i}=\mathbf{p}_{i}-\langle\mathbf{p}\rangle$ where $\langle\mathbf{p}\rangle:=1 / N \sum_{i} \mathbf{p}_{i}$ is the average (total momentum), which thanks to symmetry is conserved in time, $\langle\dot{\mathbf{p}}\rangle(t) \propto \sum_{i j} a_{i j}\left(\mathbf{p}_{i}-\mathbf{p}_{j}\right)=0$, and hence the symmetric system (1.1) reads

$$
\frac{d}{d t} \mathbf{q}_{i}(t)=\alpha \sum_{j=1}^{N} a_{i j}\left(\mathbf{q}_{j}-\mathbf{q}_{i}\right), \quad \mathbf{q}_{i}:=\mathbf{p}_{i}-\langle\mathbf{p}\rangle .
$$

Let $L_{A}:=I-A$ denote the Laplacian matrix associated with $A$, with ordered eigenvalues $0=\lambda_{1}\left(L_{A}\right) \leq \lambda_{2}\left(L_{A}\right) \leq \ldots \lambda_{N}\left(L_{A}\right)$. The following estimate is at the heart of matter (here $|\cdot|$ denotes the usual Euclidean norm on $\mathbb{R}^{d}$ ),

$$
\frac{1}{2} \frac{d}{d t} \sum_{i}\left|\mathbf{q}_{i}(t)\right|^{2}=\alpha \sum_{i, j} a_{i j}\left\langle\mathbf{q}_{j}-\mathbf{q}_{i}, \mathbf{q}_{i}\right\rangle=-\frac{\alpha}{2} \sum_{i j} a_{i j}\left|\mathbf{q}_{j}-\mathbf{q}_{i}\right|^{2} \leq-\alpha \lambda_{2}\left(L_{A}\right) \sum_{i}\left|\mathbf{q}_{i}(t)\right|^{2} .
$$

The second equality is a straightforward consequence of $A$ being symmetric; the following inequality follows from the Courant-Fischer characterization of the second eigenvalue of $L_{A}$ in 
terms of vectors $\mathbf{q}$ orthogonal to the first eigenvector $\mathbf{1}=(1,1, \ldots, 1)^{\top}$,

$$
\lambda_{2}\left(L_{A}\right)=\min _{\sum \mathbf{q}_{k}=0} \frac{\left\langle L_{A} \mathbf{q}, \mathbf{q}\right\rangle}{\langle\mathbf{q}, \mathbf{q}\rangle} \leq \frac{(1 / 2) \sum_{i j} a_{i j}\left|\mathbf{q}_{i}-\mathbf{q}_{j}\right|^{2}}{\sum_{i}\left|\mathbf{q}_{i}\right|^{2}} .
$$

We end up with the following sufficient condition for the emergence of unconditional concentration.

Theorem 2.6 (Unconditional concentration in the symmetric case). Consider the self-organized model (1.1), 2.1) with a symmetric adjacency matrix $A$. Then the following concentration estimate holds

$$
\vee_{\mathbf{p}(t)} \leq \exp \left(-\alpha \int^{t} \lambda_{2}\left(L_{A(\mathcal{P}(s))}\right) d s\right) \vee_{\mathbf{p}(0)}, \quad \vee_{\mathbf{p}(t)}^{2}:=\frac{1}{N} \sum_{i}\left|\mathbf{p}_{i}(t)-\langle\mathbf{p}\rangle(0)\right|^{2} .
$$

In particular, if the interactions remain "sufficiently strong" so that $\int^{\infty} \lambda_{2}\left(L_{A(\mathcal{P}(s))}\right) d s=\infty$, then there is convergence towards consensus $\mathbf{p}_{i}(t) \rightarrow \mathbf{p}^{\infty}=\langle\mathbf{p}\rangle(0)$.

To apply theorem 2.6, we need to trace effective lower bounds on $\lambda_{2}\left(L_{A}\right)$; here are two examples which recover our previous results in section 2.1.

Example \# $\mathbf{1}$ (revisiting theorem 2.2). If $\mathbf{r}$ is the Fiedler eigenvector associated with $\lambda_{N-1}(A)$ with $\mathbf{r} \perp \mathbf{1}$, then (2.7) implies

$$
\lambda_{N-1}(A)=\frac{[A \mathbf{r}]}{[\mathbf{r}]} \leq \sup _{\mathbf{p} \perp \mathbf{1}} \frac{[A \mathbf{p}]}{[\mathbf{p}]} \leq 1-\eta_{A} .
$$

We end up with the following lower bound for the Fiedler number

$$
\lambda_{2}\left(L_{A}\right)=1-\lambda_{N-1}(A) \geq 1-\left(1-\eta_{A}\right) \geq \eta_{A} .
$$

Thus, theorem 2.2 is recovered here as a special case of the sharp bound 2.20 in theorem 2.6. The former has the advantage that it applies to non-symmetric models, but as remarked earlier, is limited to models with global interactions; the latter can address the consensus of local, connected models, consult section 6 below.

We remark in passing that while theorem 2.2 employs the $\ell^{\infty}$-based diameter, $[\mathbf{p}]=[\mathbf{p}]_{\infty}=$ $\max _{i j}\left|\mathbf{p}_{i}-\mathbf{p}_{j}\right|$, then theorem 2.6 is in fact the corresponding $\ell^{2}$-based diameter, $[\mathbf{p}]_{2}^{2}:=$ $\sum_{i j}\left|\mathbf{p}_{i}-\mathbf{p}_{j}\right|^{2} /(2 N)=\vee_{\mathbf{p}}$

Example \#2 (revisiting propositions 2.4 and 2.5). A straightforward lower bound $\lambda_{2}\left(L_{A}\right) \geq$ $N$ min $a_{i j}$ recovers corollary 2.3 ,

$$
\vee_{\mathbf{p}(t)} \leq \exp \left(-\alpha \int^{t} m(s) d s\right) \vee_{\mathbf{p}(0)}, \quad m(t):=\min _{i j} \phi\left(\left|\mathbf{x}_{i}(t)-\mathbf{x}_{j}(t)\right|\right),
$$

The characterization of concentration in theorem 2.6 is sharp in the sense that the estimate (2.18) is. Indeed, it is well known that positivity of the Fiedler number, $\lambda_{2}\left(L_{A}\right)>0$, characterizes the algebraic connectivity of the graph associated with the adjacency matrix $A$, [53, 87, 29]. Theorem 2.6 places a minimal requirement on the amount of connectivity as a necessary condition for consensus 1 . There are many characterizations for the algebraic connectivity of static graphs [29, 45, 53, 54, 56, 86, 87, 95]. In the present context of self-organized dynamics (1.1), however, the dynamics of $\dot{\mathbf{p}}=\alpha(A \mathbf{p}-\mathbf{p})$ dictates the connectivity of $A=A(\mathcal{P}(t))$, which in

\footnotetext{
${ }^{1}$ We ignore possible cases in which the self-organized dynamics may regain connectivity under "cluster dynamics", namely, agents separated into disconnected clusters and merging into each other at a later stage.
} 
turn, determines the clustering behavior of the dynamics, due to the nonlinear dependence, $A=A(\mathcal{P}(t))$. Thus, the intricate aspect of the self-organized dynamics (1.1) is tracing its algebraic connectivity over time through the self-propelled mechanism in which the nonlinear dynamics and algebraic connectivity are tied together. This issue will be explored in the next sections, dealing with clustering driven by local interactions.

\section{LOCAL INTERACTIONS AND CLUSTERING}

In this section we consider the self-organized dynamics (1.1) of a "crowd" of $N$ agents, $\mathcal{P}=\left\{\mathbf{p}_{i}\right\}_{i=1}^{N}$ which does not interact globally: entries in their adjacency matrix may vanish, $a_{i j} \geq 0$. The dynamics is dictated by local interactions and its large time behavior leads to the formation of one or more clusters.

3.1. The formation of clusters. A cluster $\mathcal{C}$ is a connected subset of agents, $\left\{\mathbf{p}_{i}\right\}_{i \in \mathcal{C}}$, which is separated from all other agents outside $\mathcal{C}$, namely

$\# 1 . \quad a_{i j} \neq 0$ for all $i, j \in \mathcal{C}$; $\quad$ and $\# 2 . \quad a_{i j}=0$ whenever $i \in \mathcal{C}$ and $j \notin \mathcal{C}$.

The important feature of such clusters is their self-contained dynamics in the sense that

$$
\frac{d}{d t} \mathbf{p}_{i}=\alpha \sum_{j \in \mathcal{C}} a_{i j}\left(\mathbf{p}_{j}-\mathbf{p}_{i}\right), \quad \sum_{j \in \mathcal{C}} a_{i j}=1, \quad i \in \mathcal{C} .
$$

The dynamics of such self-contained clusters is covered by the concentration statements of global dynamics in section 2. In particular, if cluster $\mathcal{C}(t)$ remains connected and isolated for sufficiently long time, then its agents will tend to concentrate around a local consensus,

$$
\mathbf{p}_{i}(t) \stackrel{t \rightarrow \infty}{\longrightarrow} \mathbf{p}_{\mathcal{C}}^{\infty}, \text { for all } i \in \mathcal{C} .
$$

The intricate aspect, however, is the last if statement: the evolution of agents in a cluster $\mathcal{C}$ may become influenced by non- $\mathcal{C}$ agents, and in particular, different clusters may merge over time.

In the following, we fix our attention on the particular models for opinion and flocking dynamics, expressed in the unified framework (1.4),

$$
\frac{d}{d t} \mathbf{p}_{i}=\alpha \sum_{j=1}^{N} a_{i j}\left(\mathbf{p}_{j}-\mathbf{p}_{i}\right), \quad a_{i j}=a_{i j}(\mathbf{x})=\frac{1}{\sigma_{i}} \phi\left(\left|\mathbf{x}_{i}-\mathbf{x}_{j}\right|\right) ;
$$

Recall that $\mathbf{p} \mapsto \mathbf{x}$ in opinion dynamics, $\mathbf{p} \mapsto \dot{\mathbf{x}}$ in flocking dynamics, and $\sigma_{i}$ is the degree,

$$
\begin{cases}\sigma_{i}=N, & \text { symmetric model, } \\ \sigma_{i}=\sum_{j \neq i} \phi\left(\left|\mathbf{x}_{i}-\mathbf{x}_{j}\right|\right), & \text { non symmetric model. }\end{cases}
$$

We assume that the influence function $\phi$ is compactly supported

$$
\operatorname{Supp}\{\phi(\cdot)\}=[0, R] .
$$

A cluster $\mathcal{C}=\mathcal{C}(t) \subset\{1,2, \ldots, N\}$ is dictated by the finite diameter of the influence function $\phi$ such that the following two properties hold:

$$
\text { \#1. } \max _{i, j \in \mathcal{C}(t)}\left|\mathbf{x}_{i}(t)-\mathbf{x}_{j}(t)\right| \leq R ; \quad \text { and } \quad \# 2 . \quad \min _{i \in \mathcal{C}(t), j \notin \mathcal{C}(t)}\left|\mathbf{x}_{i}(t)-\mathbf{x}_{j}(t)\right|>R \text {. }
$$

When the dynamics is global, $R \gg[\mathbf{x}(0)]$, then the whole crowd of agents can be considered as one connected cluster. Here we consider the local dynamics when $R$ is small enough relative to the active diameter of the global dynamics: $R<[\mathbf{x}(0)]$ in the opinion dynamics $(1.2)$, or 
$R<D_{\infty}$ in the flocking dynamics $(1.3)$. The statements of global concentration towards a consensus state asserted in propositions 2.4 and 2.5 do not apply. Instead, the local dynamics of agents leads them to concentrate in one or several clusters - consult for example, figures 1.1 and 3.3 , 5.2 below. Our primary interest is in the large time behavior of such clusters. The generic scenario is a crowd of agents which is partitioned into a collection of clusters, $\mathcal{C}_{k}, k=1, \ldots K$, such that

$$
\begin{cases}\text { either } & \left|\mathbf{p}_{i}(t)-\mathbf{p}_{j}(t)\right| \stackrel{t \rightarrow \infty}{\longrightarrow} 0, \quad \text { if } \quad i, j \in \mathcal{C}_{k} \leftrightarrow\left|\mathbf{x}_{i}(t)-\mathbf{x}_{j}(t)\right| \leq R \\ \text { or } \quad & \left|\mathbf{x}_{i}(t)-\mathbf{x}_{j}(t)\right|>R, \quad \text { if } \quad i \in \mathcal{C}_{k}, j \in \mathcal{C}_{\ell}, \quad k \neq \ell .\end{cases}
$$

In this context, we raise the following two fundamental questions.

Question \#1. Identify the class of initial configurations, $\mathcal{P}(0)$, which evolve into finitely many clusters, $\mathcal{C}_{k}, k=1, \ldots K$. In particular, characterize the number of such clusters $K$ for $t \gg 1$.

Question \#2. Assume that the initial configuration $\mathcal{P}(0))$ is connected. Characterize the initial configuration $\mathcal{P}(0)$ which evolve into one cluster, $K(t)=1$ for $t \gg 1$, namely, the question of emerging of consensus in the local dynamics.

A complete answer to these questions should provide an extremely interesting insight into local processes of self-organized dynamics, with many applications. In the next two sections we provide partial answers to these questions. We begin with the first result which shows that if the solution of (3.1) has bounded time-variation then it must be partitioned into a collection of clusters.

Proposition 3.1 (Formation of clusters). Let $\mathcal{P}(t)=\left\{\mathbf{p}_{k}(t)\right\}_{k}$ be the solution of the opinion or flocking models (3.1) with compactly supported influence function $\operatorname{Supp}\{\phi(\cdot)\}=[0, R)$, and assume it has a bounded time-variation

$$
\int^{\infty}\left|\dot{\mathbf{p}}_{i}(s)\right| d s<\infty
$$

Then $\mathcal{P}(t)$ approaches a stationary state, $\mathbf{p}^{\infty}$, which is partitioned into $K$ clusters, $\left\{\mathcal{C}_{k}\right\}_{k=1}^{K}$, such that $\{1,2, \ldots, N\}=\cup_{k=1}^{K} \mathcal{C}_{k}$ and

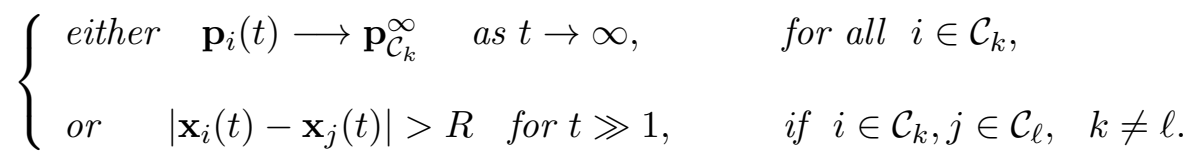

Remark. Observe that if the solution decays fast enough - in particular, if $\mathbf{p}(t)$ decays exponentially fast, $\left|\mathbf{p}_{i}(t)-\mathbf{p}_{i}^{\infty}\right| \lesssim e^{-C\left(t-t_{0}\right)}, \quad t \geq t_{0}>0$ (as in the unconditional consensus and flocking of global interactions discussed in section 21, then it has a bounded time-variation.

Proof. Assumption (3.3) implies

$$
\left|\mathbf{p}_{i}\left(t_{2}\right)-\mathbf{p}_{i}\left(t_{1}\right)\right| \leq \int_{t_{1}}^{t_{2}}\left|\dot{\mathbf{p}}_{i}(s)\right| d s \ll 1 \text { for } t_{2}>t_{1} \gg 1,
$$

hence each agent approaches its own stationary state, $\mathbf{p}_{i}(t) \stackrel{t \rightarrow \infty}{\longrightarrow} \mathbf{p}_{i}^{\infty}$. We claim that $\dot{\mathbf{p}}_{i}(t) \stackrel{t \rightarrow \infty}{\longrightarrow}$ 0 . To this end, we distinguish between the two cases of first-order opinion dynamics and second-order flocking dynamics. In opinion dynamics, $\mathbf{p} \mapsto \mathbf{x}$ : since the expression of the right 
of (3.1),

$$
\dot{\mathbf{p}}_{i}(t)=\frac{\alpha}{\sigma_{i}(t)} \sum_{j} \phi\left(\left|\mathbf{x}_{i}(t)-\mathbf{x}_{j}(t)\right|\right)\left(\mathbf{p}_{i}(t)-\mathbf{p}_{j}(t)\right), \quad \sigma_{i}(t)=\sum_{j} \phi\left(\left|\mathbf{x}_{i}(t)-\mathbf{x}_{j}(t)\right|\right),
$$

has a limit (involving $\mathbf{p}_{i}^{\infty}=\mathbf{x}_{i}^{\infty}$ ), it follows that $\lim _{t \rightarrow \infty} \dot{\mathbf{p}}_{i}(t)$ exists and by (3.3) it must be zero, $\dot{\mathbf{p}}_{i}(t) \rightarrow 0$. In the case of flocking dynamics, $\mathbf{p} \mapsto \dot{\mathbf{x}}$, and there are two types of pairs of agents $(i, j)$ : either they have the same limiting "velocity", $\mathbf{p}_{i}^{\infty}-\mathbf{p}_{j}^{\infty}=0$, and since $\phi$ is bounded,

$$
\phi\left(\left|\mathbf{x}_{i}(t)-\mathbf{x}_{j}(t)\right|\right)\left(\mathbf{p}_{i}(t)-\mathbf{p}_{j}(t)\right) \stackrel{t \rightarrow \infty}{\longrightarrow} 0 ;
$$

or - if $\mathbf{p}_{i}^{\infty}-\mathbf{p}_{j}^{\infty} \neq 0$ then,

$$
\left|\mathbf{x}_{i}^{\infty}-\mathbf{x}_{j}^{\infty}\right| \gtrsim\left|\mathbf{p}_{i}^{\infty}-\mathbf{p}_{j}^{\infty}\right| t>R, \quad t \gg 1,
$$

and hence

$$
\phi\left(\left|\mathbf{x}_{i}(t)-\mathbf{x}_{j}(t)\right|\right)\left(\mathbf{p}_{i}(t)-\mathbf{p}_{j}(t)\right)=0, \quad t \gg 1 .
$$

In either case, the expression on the right of (3.5) vanishes as $t \rightarrow \infty$.

Now, take the scalar product of (3.5) against $\mathbf{p}_{i}$ and sum,

$$
\sum_{i} \sigma_{i}\left\langle\dot{\mathbf{p}}_{i}, \mathbf{p}_{i}\right\rangle=\alpha \sum_{i j} \phi_{i j}\left\langle\mathbf{p}_{j}-\mathbf{p}_{i}, \mathbf{p}_{i}\right\rangle \equiv-\frac{\alpha}{2} \sum_{i j} \phi_{i j}\left|\mathbf{p}_{j}-\mathbf{p}_{i}\right|^{2}
$$

Since $\mathbf{p}_{i} \in \Omega(0), \sigma_{i} \leq N$ are uniformly bounded and $\dot{\mathbf{p}}_{i}(t) \rightarrow 0$ on the left, it follows that the expression on the right tends to zero. In opinion dynamics $(\mathbf{p} \mapsto \mathbf{x})$ we can pass to the limit on the expression on the right which yields

$$
\phi\left(\left|\mathbf{x}_{i}^{\infty}-\mathbf{x}_{j}^{\infty}\right|\right)\left|\mathbf{p}_{i}^{\infty}-\mathbf{p}_{j}^{\infty}\right|^{2}=0, \quad \text { for all } i, j \leq N .
$$

Thus, if $\left|\mathbf{x}_{i}^{\infty}-\mathbf{x}_{j}^{\infty}\right|>R$, then agents $i$ and $j$ are in separate clusters. Otherwise, when they are in the same cluster, say $i, j \in \mathcal{C}_{k}$ so that $\left|\mathbf{x}_{i}^{\infty}-\mathbf{x}_{j}^{\infty}\right|<R$, then $\phi\left(\left|\mathbf{x}_{i}^{\infty}-\mathbf{x}_{j}^{\infty}\right|\right)>0$ and by (3.8) they must share the same stationary state, $\mathbf{p}_{i}^{\infty}=\mathbf{p}_{j}^{\infty}=: \mathbf{p}_{\mathcal{C}_{k}}^{\infty}$, that is, 3.4 holds. In the case of flocking dynamic, $\mathbf{p} \mapsto \dot{\mathbf{x}}$, we either have one type of pairs, $\mid \mathbf{p}_{i}(t)-\mathbf{p}_{j}(t) \stackrel{t \rightarrow \infty}{\longrightarrow} 0$ or a second type of pairs, (3.6), namely, (3.4) holds.

We now turn our attention to the number of clusters, $K$.

3.2. How many clusters? Note that if $\mathbf{p}^{\infty}=\left(\mathbf{p}_{1}^{\infty}, \ldots, \mathbf{p}_{N}^{\infty}\right)^{\top}$ be a stationary state of (3.1) then $\mathbf{p}^{\infty}$ is an eigenvector associated with the nonlinear eigenvalue problem,

$$
A\left(\mathbf{x}^{\infty}\right) \mathbf{p}^{\infty}=\mathbf{p}^{\infty}
$$

corresponding to the eigenvalue $\lambda_{N}\left(A\left(\mathbf{x}^{\infty}\right)\right)=1$. Actually, the number of stationary clusters can be directly computed from the multiplicity of leading spectral eigenvalues of $\lambda_{N}\left(A\left(\mathbf{x}^{\infty}\right)\right)$.

Proposition 3.2. Assume that the crowd of $N$ agents $\left\{\mathbf{p}_{i}(t)\right\}_{i=1}^{N}$ is partitioned into $K$ clusters, $\{1,2, \ldots, N\}=\cup_{k=1}^{K(t)} \mathcal{C}_{k}$. Then, the number of clusters, $K=K(t)$, equals the geometric multiplicity of $\lambda_{N}(A(\mathbf{x}(t))=1$,

$$
K(t)=\left\{\# \lambda _ { N } \left(A(\mathbf{x}(t)) \mid \lambda_{N}(A(\mathbf{x}(t))=1\} .\right.\right.
$$


Proof. We include the rather standard argument for completeness. Suppose that the dynamics of 3.1) at time $t$ consists of $K=K(t)$ clusters, $\cup_{k=1}^{K(t)} \mathcal{C}_{k}$. Define the vector $\mathbf{r}^{k}=\left(r_{1}^{k}, \ldots, r_{N}^{k}\right)^{\top}$ such that:

$$
r_{j}^{k}=\left\{\begin{array}{ll}
1 & \text { if } j \in \mathcal{C}_{k} \\
0 & \text { otherwise }
\end{array} .\right.
$$

We obtain

$$
\left(A \mathbf{r}^{k}\right)_{i}=\sum_{j} a_{i j} r_{j}^{k}=\sum_{j \in \mathcal{C}_{k}} a_{i j}
$$

Using the fact that $A$ is a stochastic matrix and that $a_{i j}=0$ if $\mathbf{x}_{i}$ and $\mathbf{x}_{j}$ are not in the same cluster, we deduce

$$
\sum_{j \in \mathcal{C}_{k}} a_{i j}=\left\{\begin{array}{ll}
1 & \text { if } i \in \mathcal{C}_{k} \\
0 & \text { otherwise }
\end{array}\right\}=\mathbf{r}_{i}^{k}
$$

and therefore $A \mathbf{r}^{k}=\mathbf{r}^{k}$. Thus, associated with each cluster $\mathcal{C}_{k}$, there is an eigenvector $\mathbf{r}^{k}$ corresponding to $\lambda_{N}(A)=1$. To conclude the proof, we have to show that there are no other vectors $\mathbf{r}$ satisfying $A \mathbf{r}=\mathbf{r}$. Indeed, assume that $A \mathbf{r}=\mathbf{r}$,

$$
\sum_{j} a_{i j} r_{j}=r_{i} \quad \text { for any } i .
$$

Fix a cluster $\mathcal{C}_{k}$. Then for any $p \in \mathcal{C}_{k}$ we have

$$
\sum_{j \in \mathcal{C}_{k}} a_{p j} r_{j}=r_{p} \quad \text { for any } p \in \mathcal{C}_{k} .
$$

Denote by $r_{q}$ the maximal entry of $r_{j}$ 's on the left, corresponding to some $q \in \mathcal{C}_{k}$ : since $\sum_{j \in \mathcal{C}_{k}} a_{p j}=1$ with $a_{p j}>0$, we deduce that for any $p \in \mathcal{C}_{k}$ we have $r_{p}=\sum a_{p j} r_{j} \leq \sum a_{p j} r_{q}=$ $r_{q}$. Thus, the entries of $\mathbf{r}$ are constant on the cluster $\mathcal{C}_{k}$, so that $\mathbf{r} \propto \mathbf{r}^{k}$.

3.3. Numerical simulations with local dynamics. We illustrate the emergence of clusters with one- and two-dimensional simulations of the opinions dynamics model $1.2 \mathrm{~b}$,

$$
\frac{d}{d t} \mathbf{x}_{i}=\sum_{j} \frac{\phi_{i j}}{\sum_{k} \phi_{i k}}\left(\mathbf{x}_{j}-\mathbf{x}_{i}\right), \quad \mathbf{x}_{i}(t) \in \mathbb{R}^{d} .
$$

The influence function, $\phi$, was taken as the characteristic function of the interval $[0,1]: \phi(r)=$ $\chi_{[0,1]}$, and we use the Runge-Kutta method of order 4 with a time step of $\Delta t=.05$, for the time discretization of the system of ODEs (3.10).

As a first example, we run a simulation of the one-dimensional opinion model, $d=1$, subject to initial configuration of $N=100$ agents uniformly distributed on the interval $[0,10]$. In the figure 3.1 (Left), we plot the evolution of the opinions $\mathbf{x}_{i}(t)$ in time. We observe the formation of 4 clusters after 15 unit time. The histogram of the distribution of agents at the final time $t=40$ (figure 3.1 right) shows that the distance between the clusters is greater than 1 as predicted by proposition 3.1. We also observe that the number of opinions contained in each cluster differs (respectively 35, 14, 31 and 20 agents). Indeed, the larger cluster at $x \approx 2$ with 35 opinions is a merge between 3 branches (figure 3.1) with one branch in the middle connecting the two external branches. When the two external branches finally connect at $t \approx 8.5$ (their distance is less than 1), we observe an abrupt change in the dynamics following by a merge of the 3 branches into a single cluster.

To analyze the cluster formation, we also look at the evolution of the eigenvalues of the matrix of interaction $A(\mathbf{x}(t))$ in (3.10), $a_{i j}=\phi_{i j} / \sum_{k} \phi_{i k}$. In the figure 3.2, we represent the 

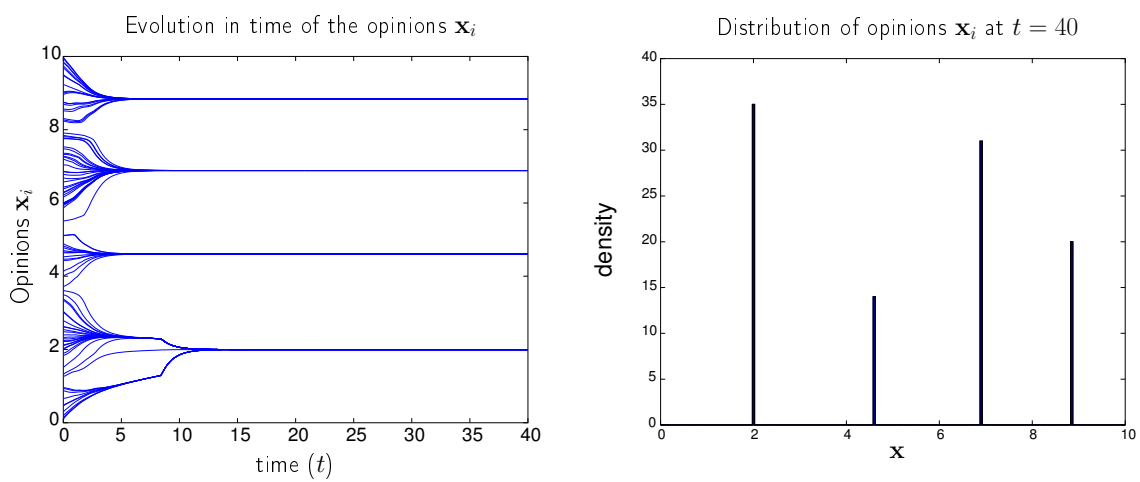

Figure 3.1. The opinion model $1.2 \mathrm{~b}$ with $M=100$ agents and $\phi=\chi_{[0,1]}$ (Left figure) and the histogram of the distribution of $\mathbf{x}_{i}$ at $t=40$ unit time (Right figure). We observe the formation of 4 clusters separated by a distance greater than 1 .

evolution of the 8 first eigenvalues of the matrix $A$. From $t=0$ to $t \approx 3.5$, we observe that the 4 first eigenvalues converge to 1 which counts for the fact that only 4 clusters remains at this time. Then the matrix $A(\mathbf{x}(t))$ remains constant in time from $t \approx 3.5$ to $t \approx 8.5$. At $t \approx 8.5$, two branches (see figure 3.1) re-connect, and the two eigenvalues $\lambda_{5}$ and $\lambda_{6}$ equal zero. This confirms proposition 3.2 where the additional multiplicity of the spectral eigenvalue $\lambda_{N}(A(\mathbf{x}(t))=1$, indicates the formation of a new cluster.

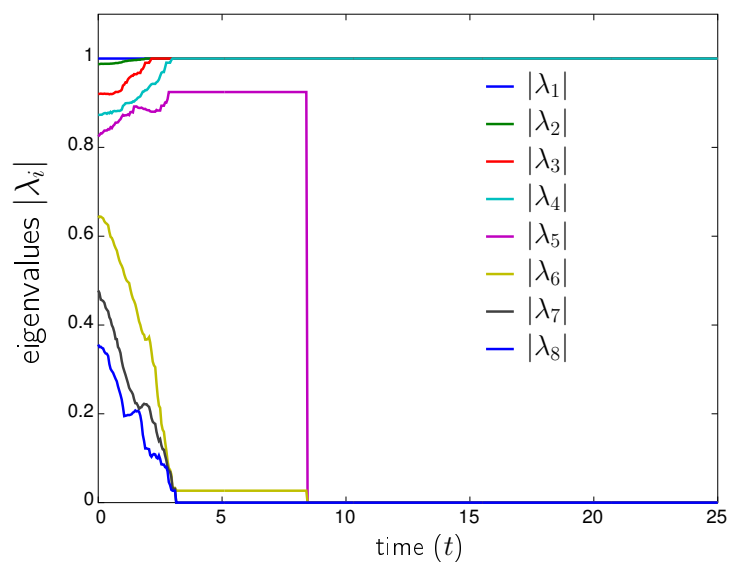

Figure 3.2. Absolute values of the eigenvalues of the matrix $A 3.10$ during the simulation given in figure 3.1. The number of eigenvalues equal to 1 corresponds to the number of clusters.

Next we turn to illustrate the dynamics of the two-dimensional, $d=2$, opinion model $(1.2 \mathrm{~b})$. With this aim, we run the model starting with an initial condition of $N=1000$ agents distributed uniformly on the square $[0,10] \times[0,10]$. We present, in figure 3.3 , several snapshots of the simulations at different time $(t=0,2,4,6,12$ and 30 unit time). As in the $1 \mathrm{D}$ case, we first observe a fast transition to a cluster formation (from $t=0$ to $t=6$ ). However at time $t=12$, the dynamics does not have yet converged to a stationary state, we observe at the upper-left that three branches are at distance less than 1 . This scenario is similar to the one observed in figure 3.1 with the apparition of 3 branches. At $t=30$, the three clusters at 
the upper left have finally merged and the system has reached a stationary state: each cluster it at distance greater than 1 from each other.

\section{4. $K=1$ : UNIFORM CONNECTIVITY IMPLIES CONSENSUS}

The emergence of a consensus in the opinion or flocking models (1.4) implies that the underlying graph associated with the dynamics must remain connected, namely $\left|\mathbf{x}_{i}(t)-\mathbf{x}_{i}(t)\right| \ll$ $R$ at least for $t \gg 1$. In this section we discuss the converse statement, namely, that uniform connectivity implies consensus. The implication of consensus in the symmetric case is based on a straightforward application of algebraic connectivity and is outlined in section 4.1. The corresponding question of consensus in non-symmetric connected models is carried out in section 4.2 using an energy method. We emphasize that consensus in both cases depend on the time-dependent behavior of intensity of connectivity, beyond the mere graph connectivity. Recall that the graph associated with $(1.1), \mathcal{G}_{A}:=(\mathcal{P}, A(\mathcal{P}))$, is connected if every two agents $\mathbf{p}_{i}(t)$ and $\mathbf{p}_{j}(t)$ are connected through a path $\Gamma_{i j}:=\left\{k_{1}=i<k_{2}<\ldots<k_{r}=j\right\}$ of length $r_{i j} \leq N$. We measure the uniform connectivity by its "weakest link".

Definition (Uniform connectivity). The self-organized dynamics 1.1) is connected if there exists $\mu(t)>0$ such that for all paths $\Gamma_{i j}$,

$$
\min _{k_{\ell} \in \Gamma_{i j}} a_{k_{\ell}, k_{\ell+1}}(\mathcal{P}(t)) \geq \mu(t)>0, \quad \text { for all } i, j .
$$

In particular, if $\mu(t) \geq \mu>0$ then we stay that $\mathcal{P}(t)$ is uniformly connected.

Alternatively, uniform connectivity of (1.1) requires the existence of $\mu=\mu_{A}>0$ independent of time, such that

$$
\left(A^{N}(\mathcal{P}(t))\right)_{i j} \geq \mu^{N}>0 .
$$

4.1. Consensus in local dynamics - symmetric models. We consider the symmetric dynamics (1.1) with associated graph $\mathcal{G}_{A}:=(\mathcal{P}, A(\mathcal{P}))$. Fix the positions of any two agents $\mathbf{p}_{i}(t)$ and $\mathbf{p}_{j}(t)$ and their (shortest) connecting path $\Gamma_{i j}$ of length $r_{i j}$. Thus, $r_{i j}$ measures the degree of separation between agents $(i, j)$, and if we let the maximal degree of separation denote the diameter of the graph, $\operatorname{diam}\left(\mathcal{G}_{A}\right):=\max _{i j} r_{i j}$, then

$$
\left|\mathbf{p}_{i}-\mathbf{p}_{j}\right|^{2} \leq \operatorname{diam}\left(\mathcal{G}_{A}\right) \sum_{k_{\ell} \in \Gamma_{i j}}\left|\mathbf{p}_{k_{\ell+1}}-\mathbf{p}_{k_{\ell}}\right|^{2}, \quad \operatorname{diam}\left(\mathcal{G}_{A}\right) \leq N .
$$

By uniform connectivity $\mu \leq a_{k_{\ell+1}, k_{\ell}}$ along each path and hence

$$
\frac{\mu}{\operatorname{diam}\left(\mathcal{G}_{A}\right)}\left|\mathbf{p}_{i}-\mathbf{p}_{j}\right|^{2} \leq \sum_{k_{\ell} \in \Gamma_{i j}} a_{k_{\ell+1}, k_{\ell}}\left|\mathbf{p}_{k_{\ell+1}}-\mathbf{p}_{k_{\ell}}\right|^{2} \leq \sum_{i j} a_{i j}\left|\mathbf{p}_{i}-\mathbf{p}_{j}\right|^{2},
$$

and summation over all pairs yields

$$
\frac{\mu}{\operatorname{diam}\left(\mathcal{G}_{A}\right)} \sum_{i j}\left|\mathbf{p}_{i}-\mathbf{p}_{j}\right|^{2} \leq N^{2} \sum_{i j} a_{i j}\left|\mathbf{p}_{i}-\mathbf{p}_{j}\right|^{2} .
$$

Now we recall our notation $\mathbf{q}_{i}:=\mathbf{p}_{i}-\langle\mathbf{p}\rangle$ : invoking 2.19$)$ we find,

$$
\lambda_{2}\left(L_{A}\right)=\min _{\sum \mathbf{q}_{k}=0} \frac{\left\langle L_{A} \mathbf{q}, \mathbf{q}\right\rangle}{\langle\mathbf{q}, \mathbf{q}\rangle}=\min _{\mathbf{p}} \frac{(1 / 2) \sum_{i j} a_{i j}\left|\mathbf{p}_{i}-\mathbf{p}_{j}\right|^{2}}{(1 / 2 N) \sum_{i j}\left|\mathbf{p}_{i}-\mathbf{p}_{j}\right|^{2}} \geq \frac{\mu}{\operatorname{Niam}\left(\mathcal{G}_{A}\right)} .
$$

Thus, the scaled connectivity factor $\mu /\left(N \operatorname{diam}\left(\mathcal{G}_{A}\right)\right) \geq \mu / N^{2}$ serves as a lower bound for the Fiedler number associated with the symmetric dynamics of (1.1) (counting the number of 

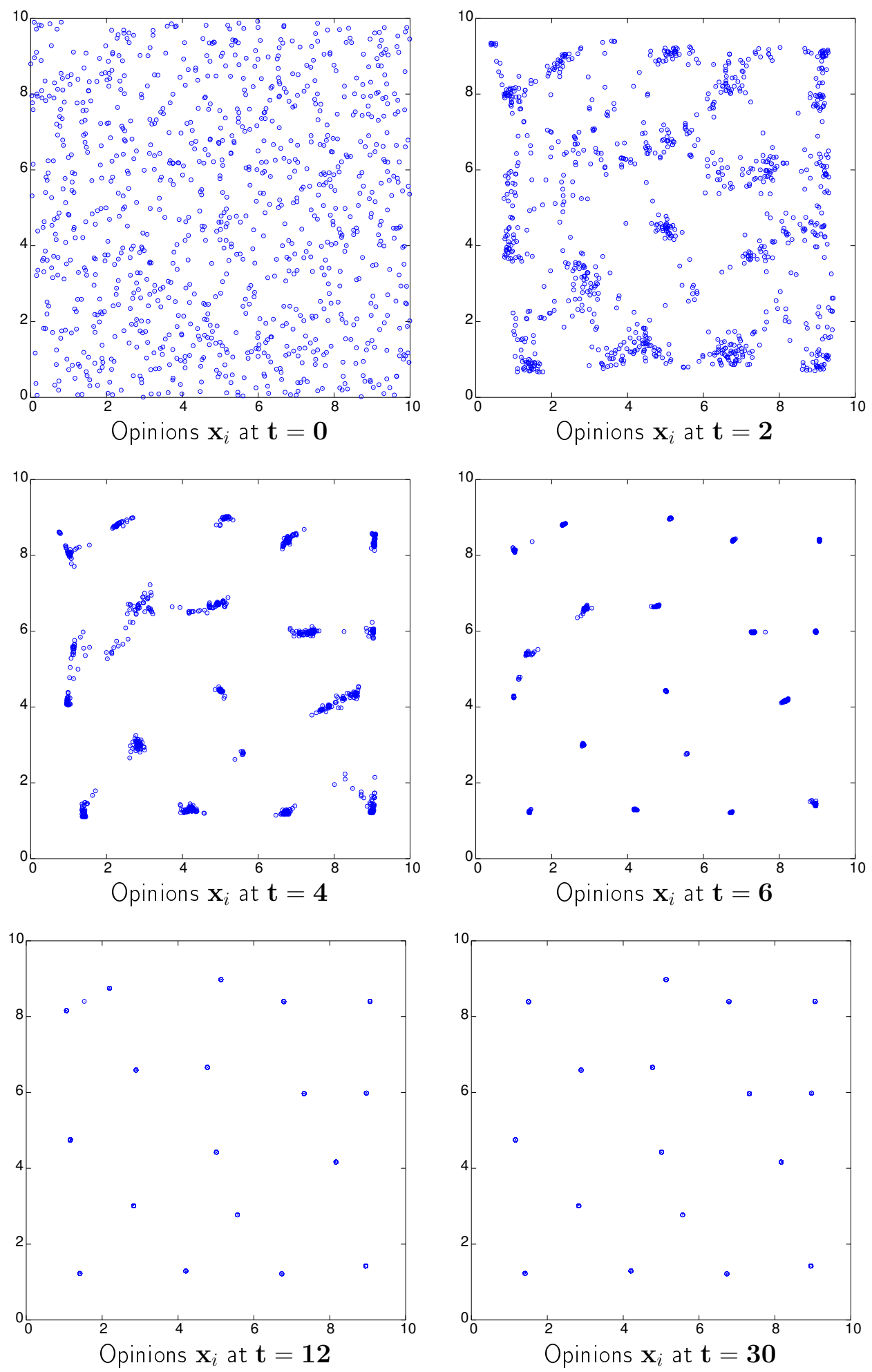

Figure 3.3. Simulation of the opinion model $(1.2 \mathrm{~b})$ in $2 \mathrm{D}$ with $M=1000$ agents and $\phi=\chi_{[0,1]}$. The dynamics converges to a cluster formation (17 clusters) with each cluster separated by a distance greater than 1 . 
"maximal" edges, yields the slightly sharper lower bound $\lambda_{2} \geq 4 \mu / N^{2}$, [87]).

Using theorem 2.6 we conclude the following.

Theorem 4.1 (Connectivity implies consensus: the symmetric case). Let $\mathcal{P}(t)=\left\{\mathbf{p}_{k}(t)\right\}_{k}$ be the solution of a symmetric self-organized dynamics

$$
\frac{d}{d t} \mathbf{p}_{i}(t)=\alpha \sum_{j \neq i} a_{i j}(\mathcal{P}(t))\left(\mathbf{p}_{j}(t)-\mathbf{p}_{i}(t)\right), \quad a_{i j}=a_{j i} .
$$

If $\mathcal{P}(t)$ remains connected in time with "sufficiently strong" connectivity $\mu_{A(\mathcal{P}(s))}>0$, then it approaches the consensus $\langle\mathbf{p}\rangle(0)$, namely,

$$
\vee_{\mathbf{p}(t)} \lesssim \exp \left(-\frac{\alpha}{N^{2}} \int_{0}^{t} \mu_{A(\mathcal{P}(s))} d s\right) \vee_{\mathbf{p}(0)}, \quad \vee_{\mathbf{p}(t)}^{2}:=\frac{1}{N} \sum\left|\mathbf{p}_{i}(t)-\langle\mathbf{p}\rangle(0)\right|^{2} .
$$

In particular, if $\mathcal{P}(t)$ remains uniformly connected in time, 4.1), then it approaches an emerging consensus, $\mathbf{p}_{i}(t) \rightarrow \mathbf{p}^{\infty}=\langle\mathbf{p}\rangle(0)$ with a convergence rate,

$$
\vee_{\mathbf{p}(t)} \lesssim e^{-\alpha \frac{\mu}{N^{2}} t} \vee_{\mathbf{p}(0)}
$$

It is important to notice that theorem 4.1 requires the intensity of connectivity to be sufficiently strong: connectivity alone, with a rapidly decaying $\mu(t)$, is not sufficient for consensus as illustrated by the following.

Counterexample. Consider the symmetric dynamics $1.2 \mathrm{a}$ with 5 agents, $x_{1}, \ldots, x_{5}$, subject to initial configuration

$$
\mathbf{x}_{1}(0)=-\mathbf{x}_{5}(0), \quad \mathbf{x}_{2}(0)=-\mathbf{x}_{4}(0), \quad \mathbf{x}_{3}(0)=0,
$$

with $\left(\mathbf{x}_{4}(0), \mathbf{x}_{5}(0)\right)$ to be specified below inside the box $\mathcal{D}:=\left\{\frac{1}{2}<\mathbf{x}_{4}<1<\mathbf{x}_{5}<\frac{3}{2}\right\}$. We fix the influence function $\phi(r)=(1+r)^{2}(1-r)^{2} \chi_{[0,1]}$, compactly supported on $[0,1]$; note that $\phi^{\prime}(0)=\phi^{\prime}(1)=0$. By symmetry, the initial ordering in 4.5$)$ is preserved in time. In particular, $\mathbf{x}_{3}(t) \equiv 0$, and $\left(\mathbf{x}_{4}(t), \mathbf{x}_{5}(t)\right) \mapsto(x(t), y(t))$ preserve the original ordering, $\frac{1}{2}<x(t)<1<y(t)$, the symmetric opinion dynamics (1.2a) (with $\alpha=5$ for simplicity), is reduced to

$$
\begin{aligned}
& \dot{x}=-\phi(|x|) x+\phi(|y-x|)(y-x) \\
& \dot{y}=\phi(|x-y|)(x-y)
\end{aligned}
$$

An equilibrium for the system is given by $x=y=1$. The eigenvalues of the linearized system at $(1,1)$ are $\lambda_{1}=0$ and $\lambda_{2}=-2$, therefore the equilibrium is unstable. We would like to prove that there exists an initial condition $(x(0), y(0))$ close to $(1,1)$ which converges toward this unstable equilibrium. We use for that a variant of the antifunnel theorem [68].

We study the phase portrait of the dynamical system (4.6) close to the unstable equilibrium $(1,1)$. Take $\varepsilon$ such that $0<\varepsilon<\frac{1}{2}$ and consider the 3 curves (see figure 4.1):

$$
\begin{array}{ll}
\alpha(s)=(2-s, s), \quad \beta(s)=(1, s) & \text { for } s \in(1,1+\varepsilon] \\
\gamma(s)=(2-s, 1+\varepsilon) & \text { for } s \in[1,1+\varepsilon]
\end{array}
$$

We denote by $\mathcal{D}_{\varepsilon}$ the domain enclosed by the 3 curves:

$$
\mathcal{D}_{\varepsilon}=\{2-y \leq x \leq 1,1<y \leq 1+\varepsilon\} .
$$

Notice that on the domain $\mathcal{D}_{\varepsilon}$, we have $\dot{y}<0$. Thus, given a solution of (4.6) starting on $\gamma$, there are 3 possibilities: the solution exits the domain passing through the curves $\alpha$, or it exits passing through $\beta$ or it converges to the equilibrium $(1,1)$. 

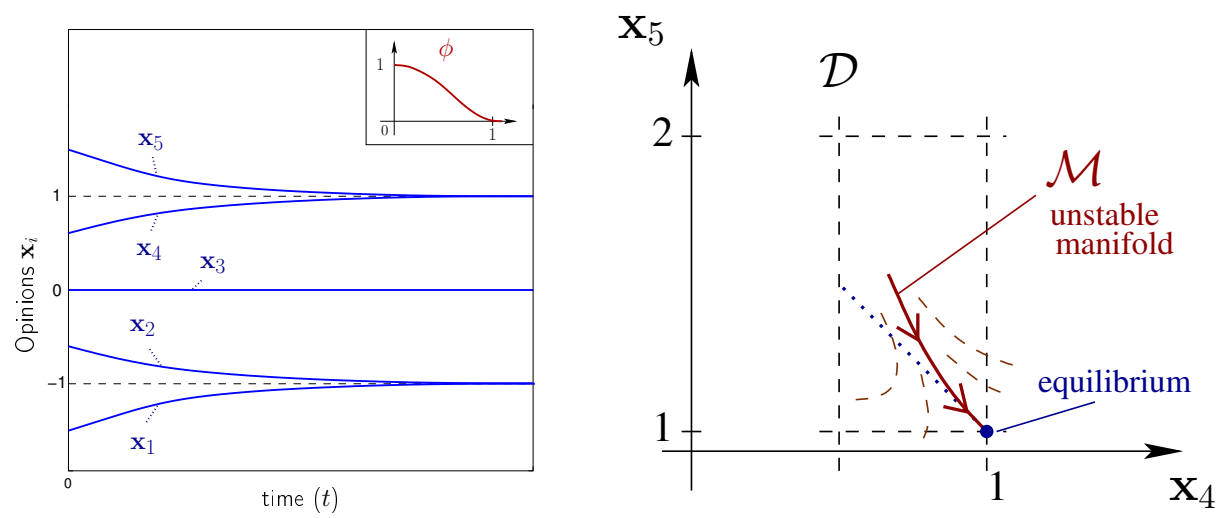

FIGURE 4.1. Left: a solution of the symmetric model that stays connected but does not converge to a consensus. Right: in phase space, the counter example is a solution that stays in the antifunnel formed by the curve $\alpha$ and $\beta$.

To prove the existence of solutions in the third category, we notice that the curves $\alpha$ and $\beta$ form an antifunnel for the dynamical system. Starting on the curve $\beta$, since $\dot{x}>0$, the solution exits the domain $\mathcal{D}_{\varepsilon}$ (see figure 4.1). Similarly, on the curve $\alpha$, since $\dot{x}<\dot{y}$, the solution exits the domain $\mathcal{D}_{\varepsilon}$ as well.

We denote by $\gamma_{\alpha}$ the set of initial conditions contained in $\gamma$ such that the solution exits through $\alpha$. The set $\gamma_{a}$ is non-empty since $(1-\varepsilon, 1+\varepsilon) \in \gamma_{a}$. Moreover, using the same arguments as in [68], we find out that $\gamma_{\alpha}$ is open. Similarly, we denote by $\gamma_{\beta} \subset \gamma$ the set of initial conditions such that the solution exits through $\beta$ and we deduce that $\gamma_{\beta}$ is open and non-empty. Since $\gamma_{\alpha} \cap \gamma_{\beta}=\emptyset$, by connectivity of the set $\gamma$, there exists $\left(x_{*}, y_{*}\right)$ which does not belong to $\gamma_{\alpha} \cup \gamma_{\beta}$. Thus, the solution $(x(t), y(t))$ starting from $\left(x_{*}, y_{*}\right)$ stays in between $\alpha$ and $\beta$ :

$$
2-y(t) \leq x(t) \leq 1 \quad, \quad 1 \leq y(t) \leq 1+\varepsilon, \quad \text { for all } t \geq 0
$$

Since $y(t)$ is decreasing and lower bounded, $y(t)$ converges: $y(t) \stackrel{t \rightarrow \infty}{\longrightarrow} y_{\infty}$. Moreover, the solution $(x(t), y(t))$ is globally Lipschitz, thus $\dot{y}$ converges to zero. Then, combining (4.6) with $\phi(|y(t)-x(t)|) \geq m>0$, we deduce that $x(t) \stackrel{t \rightarrow \infty}{\longrightarrow} y_{\infty}$. Since there is only one equilibrium in the domain $\overline{\mathcal{D}}_{\varepsilon}$, we necessarily have $y_{\infty}=1$, and therefore $(x(t), y(t) \stackrel{t \rightarrow \infty}{\longrightarrow}(1,1)$.

4.2. Consensus in local non-symmetric opinion dynamics. Next we turn to consider the question of consensus for the non-symmetric opinion model $1.2 \mathrm{~b})$.

Theorem 4.2 (Connectivity implies consensus: non-symmetric opinion dynamics). Let $\mathcal{P}(t)=$ $\left\{\mathbf{p}_{k}(t)\right\}_{k}$ be the solution of the non-symmetric opinion dynamics $1.2 \mathrm{~b}$ with compactly supported influence function, $\operatorname{Supp}\{\phi(\cdot)\}=[0, R)$,

$$
\sigma_{i} \frac{d}{d t} \mathbf{x}_{i}(t)=\alpha \sum_{j} \phi_{i j}\left(\mathbf{x}_{i}(t)-\mathbf{x}_{j}(t)\right), \quad \sigma_{i}=\sum_{k} \phi_{i k}
$$


If $\mathcal{P}(t)$ remains uniformly connected in time in the sense that each pair of agents $(i, j)$ is connected through a path $\Gamma_{i j}$ such that ${ }^{2}$

$$
\min _{k_{\ell} \in \Gamma_{i j}} \phi\left(\left|\mathbf{x}_{k_{\ell}}-\mathbf{x}_{k_{\ell+1}}\right|\right) \geq \mu>0, \quad \text { for all } i, j,
$$

then it has bounded time-variation and consequently, $\mathcal{P}(t)$ approaches an emerging consensus, $\mathbf{x}_{i}(t) \rightarrow \mathbf{x}^{\infty}$ with a convergence rate,

$$
\left|\mathbf{x}_{i}(t)-\mathbf{x}^{\infty}\right| \lesssim e^{-\alpha m\left(t-t_{0}\right)}[\mathbf{x}(0)], \quad m=\min _{r \leq R / 2} \phi(r)>0 .
$$

Proof. We introduce the energy functional,

$$
\mathcal{E}(t):=\alpha \sum_{i, j} \Phi\left(\left|\mathbf{x}_{j}(t)-\mathbf{x}_{i}(t)\right|\right), \quad \Phi(r):=\int_{s=0}^{r} s \phi(s) d s,
$$

which is decreasing in time,

$$
\begin{aligned}
\frac{d}{d t} \mathcal{E}(t) & =\alpha \sum_{i, j} \phi_{i j}\left\langle\dot{\mathbf{x}}_{j}-\dot{\mathbf{x}}_{i}, \mathbf{x}_{j}-\mathbf{x}_{i}\right\rangle=-2 \alpha \sum_{i, j} \phi_{i j}\left\langle\dot{\mathbf{x}}_{i}, \mathbf{x}_{j}-\mathbf{x}_{i}\right\rangle \\
& =-2 \sum_{i}\left\langle\dot{\mathbf{x}}_{i}, \alpha \sum_{j \neq i} \phi_{i j}\left(\mathbf{x}_{j}-\mathbf{x}_{i}\right)\right\rangle=-2 \sum_{i} \sigma_{i}\left|\dot{\mathbf{x}}_{i}\right|^{2} \leq 0
\end{aligned}
$$

To upperbound the expression on the right of 4.8a, sum (1.2b) against $\mathbf{x}_{i}$ to find,

$$
\begin{aligned}
\frac{\alpha}{2} \sum_{i, j} \phi_{i j}\left|\mathbf{x}_{i}-\mathbf{x}_{j}\right|^{2} & =-\alpha \sum_{i, j} \phi_{i j}\left\langle\mathbf{x}_{i}-\mathbf{x}_{j}, \mathbf{x}_{i}\right\rangle=\sum \sigma_{i}\left\langle\mathbf{x}_{i}, \dot{\mathbf{x}}_{i}\right\rangle \\
& \leq \sqrt{\sum_{i} \sigma_{i}\left|\mathbf{x}_{i}\right|^{2}} \sqrt{\sum_{i} \sigma_{i}\left|\dot{\mathbf{x}}_{i}\right|^{2}} \leq N \max _{i}\left|\mathbf{x}_{i}(0)\right| \sqrt{\sum_{i} \sigma_{i}\left|\dot{\mathbf{x}}_{i}\right|^{2}} .
\end{aligned}
$$

We end up with the energy decay

$$
\frac{d}{d t} \mathcal{E}(t) \leq-\frac{1}{2} \alpha^{2} C_{0}^{2}\left(\sum_{i, j} \phi_{i j}\left|\mathbf{x}_{i}-\mathbf{x}_{j}\right|^{2}\right)^{2}, \quad C_{0}=\frac{1}{N \max _{i}\left|\mathbf{x}_{i}(0)\right|}
$$

Hence, since

$$
\int^{\infty}\left(\sum_{i, j} \phi_{i j}(t)\left|\mathbf{x}_{i}(t)-\mathbf{x}_{j}(t)\right|^{2}\right)^{2} d t<\frac{2}{\alpha^{2} C_{0}^{2}} \mathcal{E}(0)<\infty,
$$

the sum $\sum_{i, j} \phi_{i j}(t)\left|\mathbf{x}_{i}(t)-\mathbf{x}_{j}(t)\right|^{2}$ must become arbitrarily small at some point of time, namely, there exists $t_{0}>0$ such that

$$
\sum_{i, j} \phi_{i j}\left(t_{0}\right)\left|\mathbf{x}_{i}\left(t_{0}\right)-\mathbf{x}_{j}\left(t_{0}\right)\right|^{2} \leq \frac{\mu}{4 N} R^{2}
$$

and by uniform connectivity, consult 4.2,

$$
\frac{\mu}{N}\left|\mathbf{x}_{i}\left(t_{0}\right)-\mathbf{x}_{j}\left(t_{0}\right)\right|^{2} \leq \sum_{k_{\ell} \in \Gamma_{i j}} \phi_{k_{\ell}, k_{\ell+1}}\left(t_{0}\right)\left|\mathbf{x}_{k_{\ell}}\left(t_{0}\right)-\mathbf{x}_{k_{\ell+1}}(t)\right|^{2} \leq \frac{\mu}{4 N} R^{2} .
$$

\footnotetext{
${ }^{2}$ Observe that here we measure connectivity in terms of the influence function $\phi_{i j}$ rather than the adjacency matrix, $a_{i j}$ as 4.1; the two are equivalent up to obvious scaling of the degree $\sigma_{i}$.
} 
Thus, the dynamics at time $t_{0}$ concentrate so that its diameter, $\left[\mathbf{x}\left(t_{0}\right)\right]=\max _{i, j} \mid \mathbf{x}_{i}\left(t_{0}\right)-$ $\mathbf{x}_{j}\left(t_{0}\right) \mid \leq R / 2$, and since $[\mathbf{x}(\cdot)]$ is non-increasing in time, $[\mathbf{x}(t)] \leq R / 2$ thereafter. Arguing along the lines of proposition 2.4 , we conclude that there is an exponential time decay,

$$
N a_{i j} \geq \phi\left(\left|\mathbf{x}_{i}(t)-\mathbf{x}_{j}(t)\right|\right) \geq \min _{r \leq[\mathbf{x}(t)]} \phi(r) \geq \min _{r \leq R / 2} \phi(r)=m, \quad t>t_{0},
$$

and consensus follows from corollary (2.3).

The decreasing energy functional $\mathcal{E}(t)$ can be used to estimate the first "arrival" time of concentration $t_{0}$. To this end, observe that:

$$
\Phi\left(\left|\mathbf{x}_{j}-\mathbf{x}_{i}\right|\right)=\int_{s=0}^{\left|\mathbf{x}_{j}-\mathbf{x}_{i}\right|} s \phi(s) d s \leq M \int_{s=0}^{\left|\mathbf{x}_{j}-\mathbf{x}_{i}\right|} s d s=M \frac{\left|\mathbf{x}_{j}-\mathbf{x}_{i}\right|^{2}}{2}, \quad M:=\max _{r} \phi(r) .
$$

Using the assumption of uniform connectivity, there exists $\mu>0$ and a path $\Gamma_{i j}$ such that:

$$
\left|\mathbf{x}_{j}-\mathbf{x}_{i}\right|^{2} \leq \frac{N}{\mu} \sum_{k_{\ell} \in \Gamma_{i j}} \phi_{k_{\ell}, k_{\ell+1}}\left|\mathbf{x}_{k_{\ell+1}}-\mathbf{x}_{k_{\ell}}\right|^{2} \leq \frac{N}{\mu} \sum_{i j} \phi_{i, j}\left|\mathbf{x}_{j}-\mathbf{x}_{i}\right|^{2} .
$$

Combining the last two inequalities, we can upperbound the energy $\mathcal{E}$ :

$$
\mathcal{E}=\sum_{i j} \Phi_{i j} \leq \frac{M N^{3}}{4 \mu} \sum_{i j} \phi_{i, j}\left|\mathbf{x}_{j}-\mathbf{x}_{i}\right|^{2}
$$

Hence, 4.10) implies the Riccati equation

$$
\frac{d}{d t} \mathcal{E}(t) \leq-\frac{1}{2} \alpha^{2} C_{0}^{2}\left(\frac{4 \mu}{M N^{3}} \mathcal{E}\right)^{2}=-\frac{C \mu^{2}}{N^{6}} \mathcal{E}^{2}
$$

which shows the energy decay

$$
\mathcal{E}(t) \lesssim \frac{1}{1+\frac{C \mu^{2} t}{N^{6}}}
$$

Thus, the arrival time of concentration $t_{0} 4.11$ is at most of the order of $\mathcal{O}\left(N^{7} / \mu^{3}\right)$. This bound on the first arrival time can be improved $]^{3}$

We close this section by noting the lack of a consensus proof for our non-symmetric model of flocking dynamics $(1.3 \mathrm{~b})$ is due to the lack of a proper decreasing energy functional.

\section{Heterophilious dynamics EnHANCES CONSENSUS — Simulations}

As we noted earlier, the large-time behavior of local models for self-organized dynamics depend on the details of the interactions, $\left\{a_{i j}\right\}$, and in the particular case of local models (3.1), on the profile of the compactly supported influence function $\phi$. Here we explore how the profile of $\phi$ dictates cluster formation in the opinion dynamics model $(1.2 \mathrm{~b})$. The numerical simulations presented in this section leads to the main conclusion that an increasing profile of $\phi$ reduces the number of clusters $\left\{\mathcal{C}_{k}\right\}_{k=1}^{K}$. In particular, if the profile of $\phi$ is increasing fast enough, then $K=1$; thus, heterophilious dynamics enhances the emergence of consensus.

\footnotetext{
${ }^{3}$ In fact, the energy $\mathcal{E}(t)$ decays exponentially in time.
} 
In the following, we employ a compactly supported influence function $\phi$ which is a simple step function,

$$
\phi(r)= \begin{cases}a & \text { for } r \leq \frac{1}{\sqrt{2}} \\ b & \text { for } \frac{1}{\sqrt{2}}<r \leq 1 \\ 0 & \text { for } r>1\end{cases}
$$

The essential quantity here is the ration $b / a$ which measures the balance between the influence of "far" and "close" neighbors (see figure 5.1). We initiate the opinion dynamics (1.2b) with random initial configuration $\left\{\mathbf{x}_{i}(0)\right\}_{i}$.

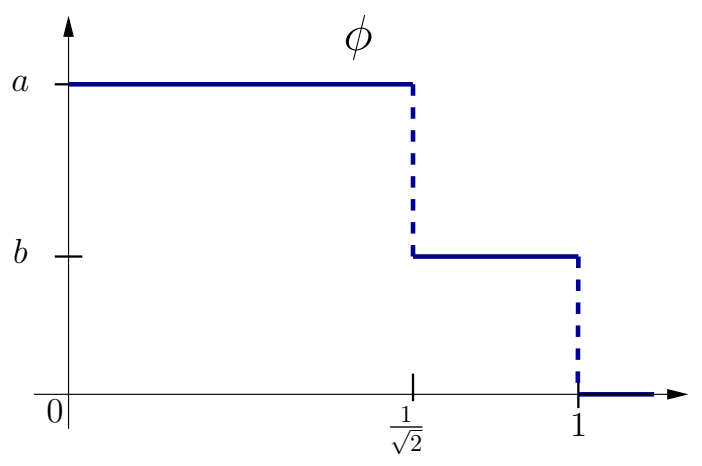

FiguRE 5.1. Influence functions $\phi$ used in the simulations. The larger $b / a$ is, the more heterophilious is the dynamics.

5.1. 1D simulations. We begin with four simulations of the $1 \mathrm{D}$ opinion dynamics $1.2 \mathrm{~b})$ subject to 100 opinions distributed uniformly on $[0,10]$, the same initial configuration as in figure 3.1. To explore the impact of the influence step function (5.1) on the dynamics, we used four different ratios of $b / a=.1,1,2$ and 10. As $b / a$ increases, we reduce the influence of the closer neighbors and increase the influence of neighbors further away; thus, increasing $b / a$ reflects the tendency to "bond with the other". As observed in figure 5.2, the increase in the ratio $b / a=.1,1,2$ and 10 , reduces the corresponding number of limit clusters to $K=6,4,2$, and for $b / a=10$, the dynamics converged to a consensus, $K=1$. The simulations of figure 5.2 indicate that reducing the influence of closer neighbors and hence increasing the weight for the influence of neighbors further away, will favor increased connectivity and the emergence of consensus.

To make a systematic analysis of the cluster formation dependence on the ratio $b / a$, we made several simulations with random initial conditions for a given ratio $b / a$. Then we make an average of the number of clusters, denoted by $\langle S\rangle$, at the end of each simulation $(t=100)$. To compute the number of clusters, we estimate the number of connected components of the matrix $A(3.10)$ using a depth-first search algorithm. As observed in figure 5.3, the number of clusters $\langle S\rangle$ decreases as $b / a$ increases. Moreover, $\langle S\rangle$ approaches 1 when $b / a$ approaches 10 , implying that a consensus is likely to occur when $b / a$ is large enough.

5.2. Clusters and branches. We revisit the opinion model $1.2 \mathrm{~b}$ with an influence step function (5.1). As noted before, the increasing value of $b / a$ increases the probability to reach a consensus. The simulations in figure 5.2 with $b / a=2$ and with $b / a=10$, show the apparition of branches, where subgroups of agents have converged to the same opinion yet, in contrast to 

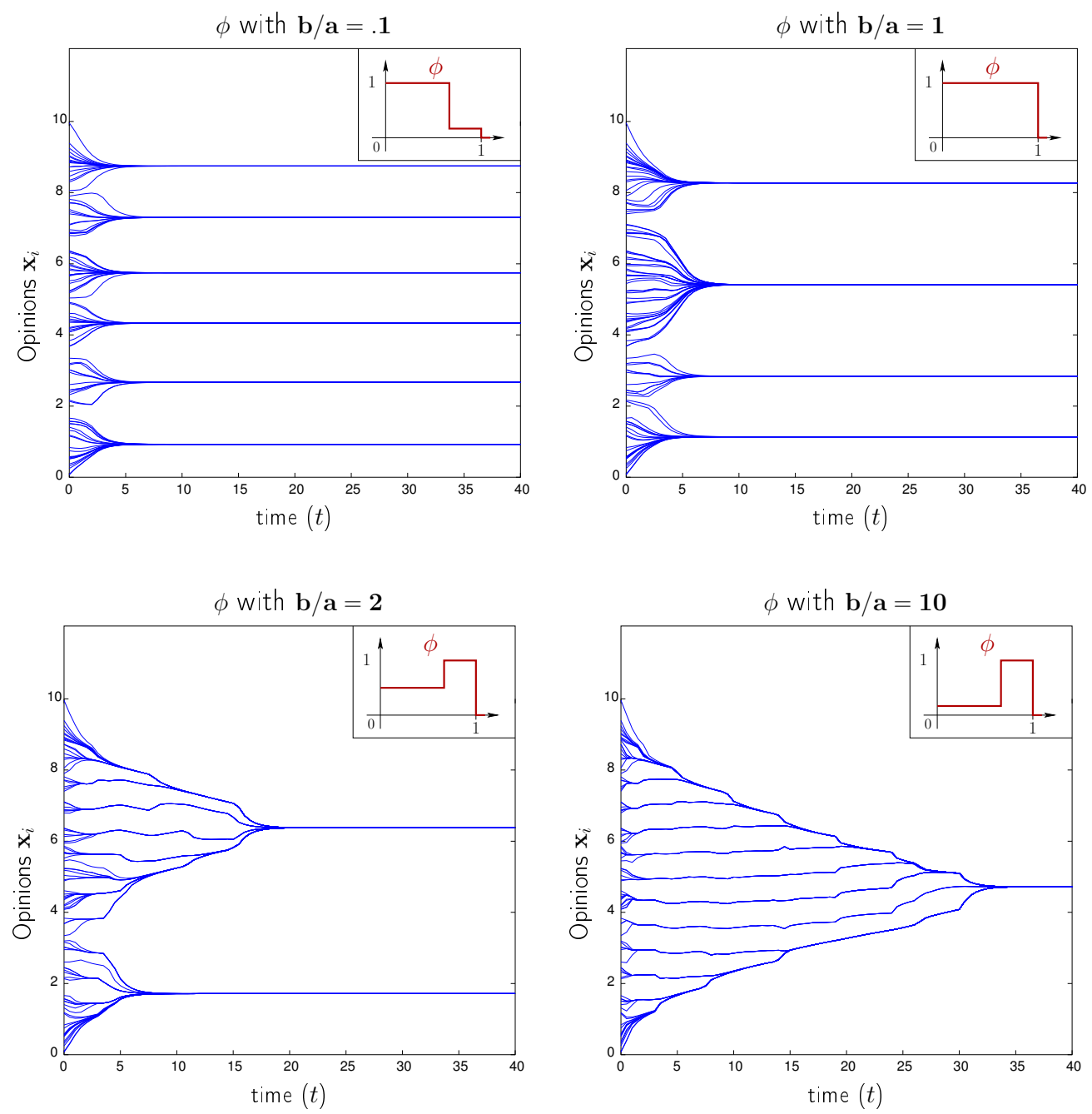

FigURE 5.2. Simulation of the opinion dynamics model with different interacting function $\phi$. When the influence of close neighbors is reduced (i.e. $b / a$ large), the number of cluster decreases. For $b / a=10$, the dynamics converges to a consensus.

clustering, these branches of opinions are still interacting with outsiders, which are in distance which is strictly less than $R=1$. In particular, when $b / a=10$, the distribution of opinions $\left\{\mathbf{x}_{i}(t)\right\}_{i}$, aggregate to form distinct branches seen in figure 5.2 at $t \sim 5$, one can identify in figure 5.4, the formation of 10 branches which are separated by a distance of approximately .7 spatial units. Since the distance between two such branches is always less than the diameter $R=1$ of $\phi$, these branches are not qualified as isolated clusters, as they continue to be influenced by "outsiders" from the nearby branches. Over time, these branches merge into each other before they emerge into one final cluster, the consensus, at $t \sim 33$. Thus, the decisive factor in the consensus dynamics is not the number of branches but their large time connected components. Indeed, figure 5.2 with $b / a=10$, shows that the agents in the different branches remain in the same connected component at distance $\sim .7$, corresponding to the discontinuity of $\phi(\cdot)$, which experience a jump from .1 to 1 at $1 / \sqrt{2} \approx .7$. 

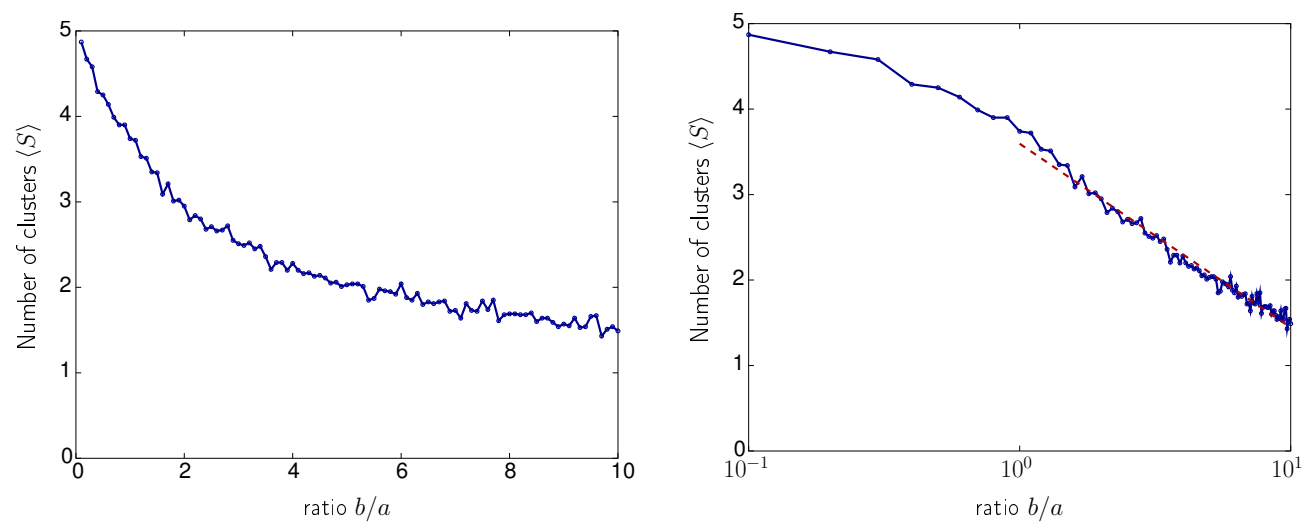

FigURE 5.3. Average number of clusters $\langle S\rangle$ depending on the ratio $b / a$ (Left figure). The larger $b / a$ is, the fewer the number of clusters. The decay is logarithmic on $[1,10]$ (Right figure). For each value of $b / a$, we run 100 simulations to estimate the mean number of clusters $\langle S\rangle$. Simulations are run with $\Delta t=.05$ and a final time equals to $t=100$ unit time.

To illustrate the apparition of the distance $1 / \sqrt{2}$ between two nearest branches, we repeat the simulations, this time with special initial configurations where all the opinions are uniformly spaced with $\left|\mathbf{x}_{i+1}-\mathbf{x}_{i}\right|=d_{*}$ with $0<d_{*}<1$. As we observe in figure 5.5, the agents $\left\{\mathbf{x}_{i}\right\}_{i}$ readjust their "opinion" such that the distance between nearest neighbors $\left|\mathbf{x}_{i+1}-\mathbf{x}_{i}\right|$ approaches $1 / \sqrt{2}$ as $t \gg 1$.

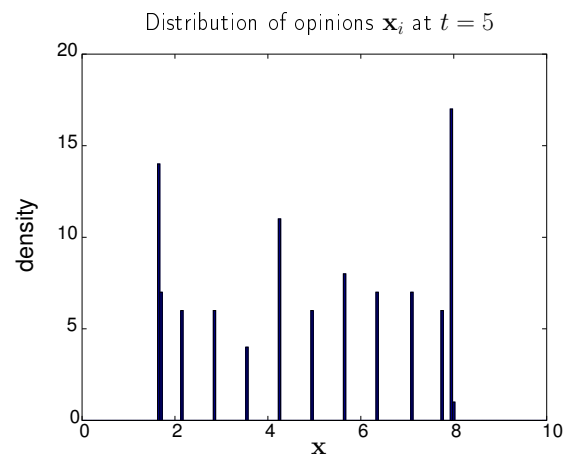

FiguRE 5.4. The distribution of $\left\{\mathbf{x}_{i}\right\}_{i}$ in the simulation of figure 5.2 with $b / a=10$ at time $t=5$. The distance between two picks of density is around $1 / \sqrt{2} \approx .7$ space unit. This distance corresponds to the discontinuity of the function $\phi(r)$.

5.3. 2D simulations. We made several 2D simulations with different influence functions $\phi$. As a first illustration, we made a $2 \mathrm{D}$ simulation with the same initial configuration used in figure 3.3, but this time we used the influence step function $\phi$ in (5.1) with $b / a=10$. In figure 5.6. one can observe a concentration phenomenon (from $t=0$ to $t=2.5$ ) - the opinions aggregate into 5 final clusters, compared with the 17 clusters observed in figure 3.3 with the influence function $\phi=\chi_{[0,1]}$. Thus, as in the $1 \mathrm{D}$ case, a more heterophilious influence function increases the clustering effect. 

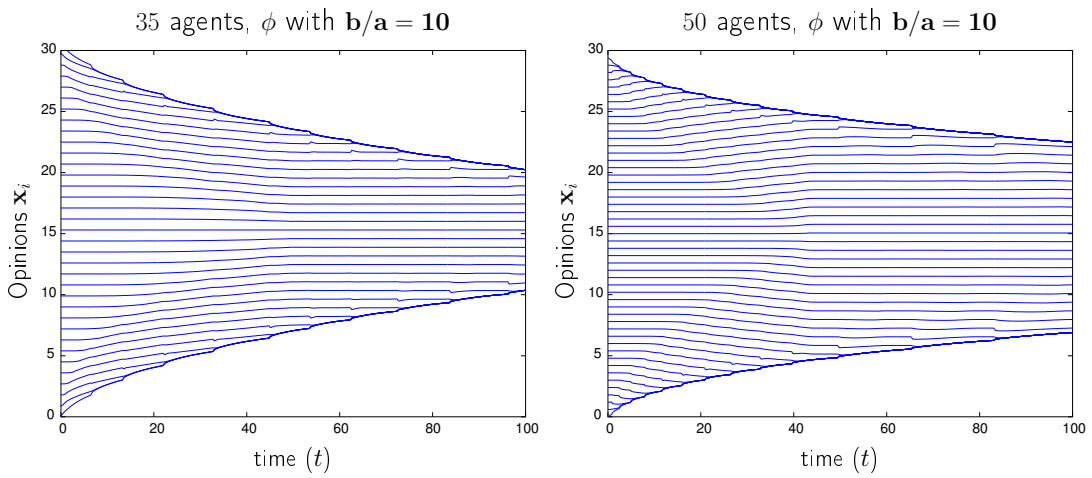

FigURE 5.5. Initial condition with an equi-repartition of $\left\{\mathbf{x}_{i}\right\}_{i}:\left|\mathbf{x}_{i+1}-\mathbf{x}_{i}\right|=.9$ (Left figure) and $\left|\mathbf{x}_{i+1}-\mathbf{x}_{i}\right|=.6$ (Right figure). Nearest neighbor readjust their distance to $1 / \sqrt{2} \approx .7$ unit space, we observe a concentration of the trajectories in the left figure and a spread of the trajectories in the right figure.

We also estimate the average number of clusters $\langle S\rangle$ depending on the ratio $b / a$. As observed in figure 5.7, $\langle S\rangle$ is a decreasing function of $b / a$ and once again the decay of $\langle S\rangle$ as a function of $b / a \in[0,10]$ is logarithmic.

\section{Heterophilious dynamics With a FiXed-Number of Neighbors}

Careful observations of startling flocks led the Rome group [25, 26, 27] to the fundamental conclusion that their dynamics is driven by local interaction with a fixed number of nearest neighbors. This motivates our study of nearest neighbor models for opinion dynamics which take the form

$$
\frac{d}{d t} \mathbf{x}_{i}=\alpha \sum_{\{j:|j-i| \leq q\}} \frac{\phi_{i j}}{\sigma_{i}}\left(\mathbf{x}_{j}-\mathbf{x}_{i}\right), \quad \mathbf{x}_{i} \in \mathbb{R}^{d},
$$

where the degree $\sigma_{i}$ is given by one of two forms, depending on the symmetric and nonsymmetric version of the opinion dynamics in 1.2

$$
\begin{cases}\text { the symmetric case : } & \sigma_{i}=\frac{1}{2 q} \\ \text { the nonsymmetric case : } & \sigma_{i}=\sum_{\{j:|j-i| \leq q\}} \phi_{i j} .\end{cases}
$$

Thus, each agent $i$ is assumed to interact only with its $2 q$ agents $i-q, \ldots, i+q$. Typically, $q$ is small (the observation in [25, 26, 27] report on six to seven active nearest neighbors). We analyze the connectivity of the particular case of two nearest neighbors, $q=1$. Here we prove that such local models preserve connectivity and hence converge to a consensus provided the influence function $\phi$ is increasing. This result supports our findings in section 5 that heterophilious dynamics is an efficient strategy to reach a consensus.

6.1. A fixed-number of neighbors with global influence function. We begin by noting that the different approaches for consensus of global models apply in the present framework of local nearest neighbor models (6.1). For example, consider the non-symmetric nearest neighbor 

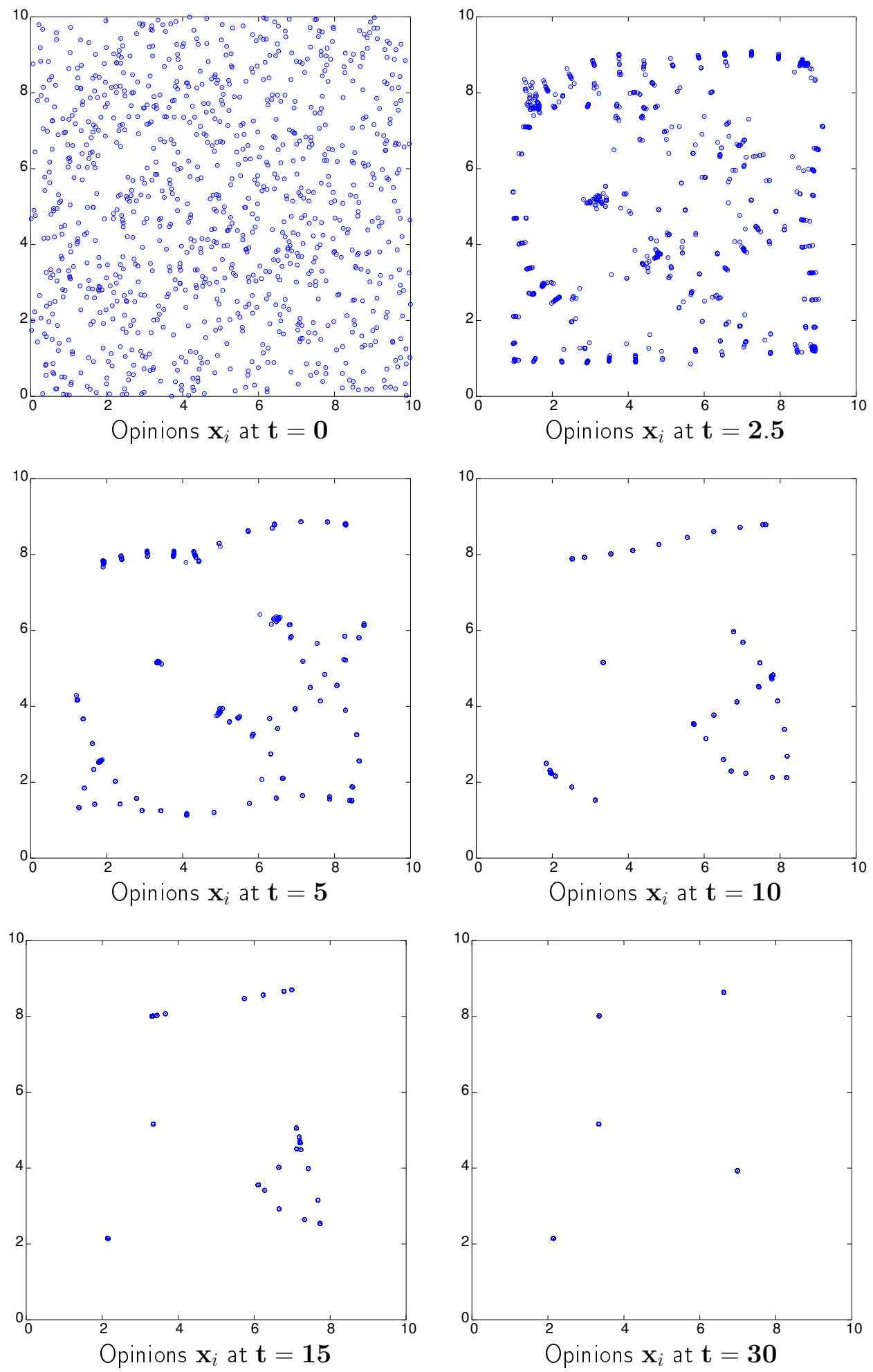

FIGURE 5.6. The heterophilious effect: diminishing the influence of close neighbors relative to those further away, increases the clustering effect. 2D simulation of the opinion model $1.2 \mathrm{~b}$ with $M=1000$ agents using a step influence function $\phi=.1 \chi_{[0,1 / \sqrt{2}]}+\chi_{[1 / \sqrt{2}, 1]}$ leads to 5 clusters which remains at the end of the simulation. This should be compared with 17 clusters with $\phi=\chi_{[0,1]}$ (see figure 3.3. 

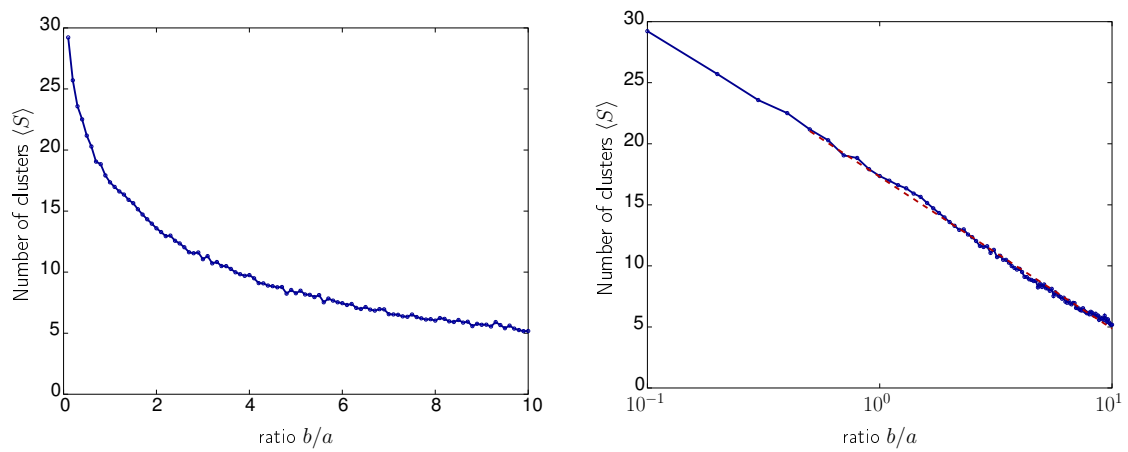

FiguRE 5.7. Average number of clusters $\langle S\rangle$ depending on the ratio $b / a$ in $2 \mathrm{D}$ (Left figure). As in $1 \mathrm{D}$ case, the larger $b / a$ is, the fewer the number of clusters, and the decay is logarithmic on $[1,10]$ (Right figure). For each value of $b / a$, we made 100 simulations to estimate the mean number of clusters $\langle S\rangle$. Simulations were made with $\Delta t=.05$ and were recorded at the final time $t=100$.

model

$$
\sigma_{i} \frac{d}{d t} \mathbf{x}_{i}=\alpha \sum_{\{j:|j-i| \leq q\}} \phi_{i j}\left(\mathbf{x}_{j}-\mathbf{x}_{i}\right), \quad \sigma_{i}=\sum_{\{j:|j-i| \leq q\}} \phi_{i j} .
$$

It admits an energy functional,

$$
\mathcal{E}(t):=\alpha \sum_{\{i, j:|i-j| \leq q\}} \Phi\left(\left|\mathbf{x}_{j}(t)-\mathbf{x}_{i}(t)\right|\right), \quad \Phi(r):=\int_{s=0}^{r} s \phi(s) d s,
$$

which is decreasing in time, $\mathcal{E}(t) \leq \mathcal{E}(0)$ and we conclude

Theorem 6.1. (Global connectivity) Consider the nearest neighbor model (6.2) with an influence function $\phi, \operatorname{Supp}\{\phi(\cdot)\}=[0, R)$ and assume $\alpha \Phi(R)>\mathcal{E}(0)$. Then $\min _{|i-j| \leq q} \phi_{i j}(t)>m_{\infty}$ where $m_{\infty}:=\min _{r<R} \phi(r)$. Hence, the nearest neighbor dynamics (6.2) remains connected and consensus follows.

Proof. Since $\mathcal{E}$ is decreasing in time,

$$
\alpha \Phi\left(\left|\mathbf{x}_{i}(t)-\mathbf{x}_{j}(t)\right|\right)<\mathcal{E}(0) \leq \alpha \Phi(R) \quad \text { for any }|i-j| \leq q,
$$

and since $\Phi(r)=\int^{r} s \phi(s) d s$ is an increasing function, $\left|\mathbf{x}_{i}(t)-\mathbf{x}_{j}(t)\right|<R$, hence $\phi_{i j}>m_{\infty}$ and consensus follows.

We note, however, that since $m_{\infty} \leq \phi \leq 1$, then $\Phi(r)$ has a quadratic bounds, $m_{\infty} r^{2} \leq$ $2 \Phi(r) \leq r^{2}$, and hence the assumption made in theorem 6.1 implies

$$
\alpha \Phi(R)>\mathcal{E}(0) \rightsquigarrow R^{2}>m_{\infty} \sum_{|i-j| \leq q}\left|\mathbf{x}_{i}-\mathbf{x}_{j}\right|^{2} .
$$

Namely, the support of $\phi$ should be sufficiently large to cover a globally connected path in phase space. 
6.2. Two-neighbor dynamics. In this section we prove uniform connectivity and hence convergence to a consensus of a symmetric two nearest neighbor model, (6.1),

$$
\frac{d}{d t} \mathbf{x}_{i}=\frac{\alpha}{2}\left(\kappa_{i+\frac{1}{2}}\left(\mathbf{x}_{i+1}-\mathbf{x}_{i}\right)+\kappa_{i-\frac{1}{2}}\left(\mathbf{x}_{i-1}-\mathbf{x}_{i}\right)\right), \quad \kappa_{i+\frac{1}{2}}:= \begin{cases}0, & i=0, N \\ \left.\phi\left(\mid \mathbf{x}_{i+1}-\mathbf{x}_{i}\right) \mid\right), & 1 \leq i \leq N .\end{cases}
$$

We assume that the initial configuration of agents can be enumerated such that $\left\{\mathbf{x}_{i}(0)\right\}_{i}$ is connected

$$
\max _{i}\left|\mathbf{x}_{i+1}(0)-\mathbf{x}_{i}(0)\right|<R, \quad \operatorname{Supp}\{\phi(\cdot)\}=[0, R) .
$$

The configuration of such "purely" local interactions applies to the one-dimensional setup where each agent is initially connected to its left and right neighbors; we emphasize that these configurations are not necessarily restricted to the one dimensional setup.

Forward differencing of $(6.3)$ implies that $\Delta_{i+\frac{1}{2}}:=\Delta_{i+\frac{1}{2}}(t):=\mathbf{x}_{i+1}(t)-\mathbf{x}_{i}(t)$ satisfy

$$
\begin{aligned}
\frac{d}{d t} \Delta_{i+\frac{1}{2}} & =\frac{\alpha}{2}\left(\kappa_{i+\frac{3}{2}}\left(\mathbf{x}_{i+2}-\mathbf{x}_{i+1}\right)+\kappa_{i+\frac{1}{2}}\left(\mathbf{x}_{i}-\mathbf{x}_{i+1}\right)-\kappa_{i+\frac{1}{2}}\left(\mathbf{x}_{i+1}-\mathbf{x}_{i}\right)-\kappa_{i-\frac{1}{2}}\left(\mathbf{x}_{i-1}-\mathbf{x}_{i}\right)\right) \\
& =\frac{\alpha}{2}\left(\kappa_{i+\frac{3}{2}} \Delta_{i+\frac{3}{2}}-2 \kappa_{i+\frac{1}{2}} \Delta_{i+\frac{1}{2}}+\kappa_{i-\frac{1}{2}} \Delta_{i-\frac{1}{2}}\right), \quad i=1,2, \ldots, N-1 .
\end{aligned}
$$

The missing $\Delta$ 's for $i=\frac{1}{2}$ and $i=N+\frac{1}{2}$ are defined as $\Delta_{\frac{1}{2}}=\Delta_{N+\frac{1}{2}}=0$. Let $\Delta_{p+\frac{1}{2}}$ denote the maximal difference, $\left|\Delta_{p+\frac{1}{2}}\right|=\max _{i}\left|\Delta_{i+\frac{1}{2}}\right|$ measured in the $\ell_{2}$-norm. Then

$$
\begin{aligned}
\frac{1}{2} \frac{d}{d t}\left|\Delta_{p+\frac{1}{2}}\right|^{2} & =\frac{\alpha}{2}\left(\kappa_{p+\frac{3}{2}}\left\langle\Delta_{p+\frac{3}{2}}, \Delta_{p+\frac{1}{2}}\right\rangle-2 \kappa_{p+\frac{1}{2}}\left|\Delta_{p+\frac{1}{2}}\right|^{2}+\kappa_{p-\frac{1}{2}}\left\langle\Delta_{p-\frac{1}{2}}, \Delta_{p+\frac{1}{2}}\right\rangle\right) \\
& \leq \frac{\alpha}{2}\left(\kappa_{p+\frac{3}{2}}-2 \kappa_{p+\frac{1}{2}}+\kappa_{p-\frac{1}{2}}\right)\left|\Delta_{p+\frac{1}{2}}\right|^{2} .
\end{aligned}
$$

Now, if $\phi$ is non-decreasing influence function, then

$$
\left|\Delta_{p+\frac{1}{2}}\right| \geq\left|\Delta_{i+\frac{1}{2}}\right| \rightsquigarrow 2 \kappa_{p+\frac{1}{2}}=2 \phi\left(\left|\Delta_{p+\frac{1}{2}}\right|\right) \geq \phi\left(\left|\Delta_{p-\frac{1}{2}}\right|\right)+\phi\left(\left|\Delta_{p+\frac{3}{2}}\right|\right),
$$

and hence $\left|\Delta_{p+\frac{1}{2}}(t)\right|=\max _{i} \phi\left(\left|\mathbf{x}_{i+1}(t)-\mathbf{x}_{i}(t)\right|\right) \leq \max _{i} \phi\left(\left|\mathbf{x}_{i+1}(0)-\mathbf{x}_{i}(0)\right|\right)$. We deduce the following theorem.

Theorem 6.2. Consider the nearest neighbor dynamics (6.3) subject to initial configuration, $\mathbf{x}(0)$ which is connected, (6.4),

$$
\max _{i}\left|\mathbf{x}_{i+1}(0)-\mathbf{x}_{i}(0)\right|<R, \quad \operatorname{Supp}\{\phi(\cdot)\}=[0, R) .
$$

Assume that the influence function, $\phi$, is non-decreasing. Then the dynamics 6.3 remains connected and converges to a consensus, $\mathbf{x}^{\infty}=\langle\mathbf{x}\rangle(0)$,

$$
\sum_{i}\left|\mathbf{x}_{i}(t)-\langle\mathbf{x}\rangle(0)\right|^{2} \lesssim \exp \left(-\frac{2 \phi(0) t}{N}\right) \sum_{i}\left|\mathbf{x}_{i}(0)-\langle\mathbf{x}\rangle(0)\right|^{2}, \quad\langle\mathbf{x}\rangle:=\frac{1}{N} \sum_{i} \mathbf{x}_{i}
$$

It is important to notice that theorem 6.2 requires an non-decreasing influence function. Indeed, the steeper the increase of $\phi$ is, the better the connectivity is. This is concrete ramification of our main statement that heterophilious dynamics enhances consensus. Note that a two-nearest neighbor dynamics driven by a decreasing $\phi$ will not guarantee consensus as illustrated by the following. 
Counterexample. We revisit the counterexample in section 4.1, of five agents symmetrically distributed around $\mathbf{x}_{3}(t) \equiv 0$ with $\frac{1}{2}<\mathbf{x}_{4}(t)<1<\mathbf{x}_{5}(t)<\frac{3}{2}$, governed by

$$
\begin{aligned}
& \dot{\mathbf{x}_{4}}=-\phi\left(\left|\mathbf{x}_{4}\right|\right) \mathbf{x}_{4}+\phi\left(\left|\mathbf{x}_{5}-\mathbf{x}_{4}\right|\right)\left(\mathbf{x}_{5}-\mathbf{x}_{4}\right), \\
& \dot{\mathbf{x}}_{5}=\phi\left(\left|\mathbf{x}_{5}-\mathbf{x}_{5}\right|\right)\left(\mathbf{x}_{4}-\mathbf{x}_{5}\right)
\end{aligned}
$$

with a compactly supported influence function $\phi(r)=(1-r)^{2}(1+r)^{2} \chi_{[0,1]}$. Observe that this configuration amounts to a two-nearest neighbor dynamics. Its concentration into three separate clusters $\{-1,0,1\}$ shown in figure 4.1, requires a rapidly decreasing influence function (to be precise - $\phi(r) r \downarrow$ for $r \sim 1$ ), which is not covered by the two-nearest neighbors' heterophilious dynamics sought in theorem 6.2 .

Proof. The adjacency matrix associated with $(6.3), \dot{\mathbf{x}}=\alpha(A \mathbf{x}-\mathbf{x})$ is given by the tridiagonal matrix $A=\left\{a_{i j}\right\}$, given by

$$
a_{i j}= \begin{cases}\frac{1}{2} \kappa_{\frac{i+j}{2}}, \quad \kappa_{\frac{i+j}{2}}=\phi\left(\left|\mathbf{x}_{i}-\mathbf{x}_{j}\right|\right) & |i-j|=1, \\ 1-\frac{1}{2} \kappa_{i}, \quad \kappa_{i}:=\phi_{i, i+1}+\phi_{i, i-1} & i=j .\end{cases}
$$

The corresponding Laplacian associated with $A$ is given by

$$
L_{A}=\frac{1}{2}\left[\begin{array}{cccccc}
-\kappa_{1} & \kappa_{\frac{3}{2}} & & & & \\
\kappa_{\frac{3}{2}} & -\kappa_{2} & \kappa_{\frac{5}{2}} & & & \\
& \kappa_{\frac{5}{2}} & -\kappa_{3} & \kappa_{\frac{7}{2}} & & \\
& & \ddots & \ddots & \ddots & \\
& & & \kappa_{N-\frac{3}{2}} & -\kappa_{N-1} & \kappa_{N-\frac{1}{2}} \\
& & & & \kappa_{N-\frac{1}{2}} & -\kappa_{N}
\end{array}\right]
$$

Since $\left|\mathbf{x}_{i+1}(t)-\mathbf{x}_{i}(t)\right|<R$, the off-diagonal entries $\kappa_{i+\frac{1}{2}}=\phi\left(\left|\mathbf{x}_{i+1}-\mathbf{x}_{i}\right|\right)>0$ and hence, the graph $\mathcal{G}_{A}=(\{\mathbf{x}(t)\}, A(\mathbf{x}(t)))$ remains connected with $\mu=\min _{i} \kappa_{i+\frac{1}{2}}$ and $\operatorname{diam}\left(\mathcal{G}_{A}\right)=N$. By (4.3) we find

$$
\lambda_{2}\left(L_{A}\right) \geq \frac{\min _{i} \kappa_{i+\frac{1}{2}}}{N^{2}} \geq \frac{\phi(0)}{N^{2}} .
$$

Using theorem 2.6 (see 2.21) $)$ we end up with

$$
\sum_{i}\left|\mathbf{x}_{i}(t)-\langle\mathbf{x}\rangle(0)\right|^{2} \lesssim \mathrm{e}^{-\frac{\alpha \phi(0) t}{N^{2}}} \sum_{i}\left|\mathbf{x}_{i}(0)-\langle\mathbf{x}\rangle(0)\right|^{2}
$$

which concludes the proof.

Remark. The worst case scenario for the decaying of the $\left|\mathbf{x}_{i}(t)-\langle\mathbf{x}\rangle\right|$ is to have many opinions $\mathbf{x}_{i}$ concentrate at two extreme values with just one path of opinion connecting the two extremes (see figure 6.1). 


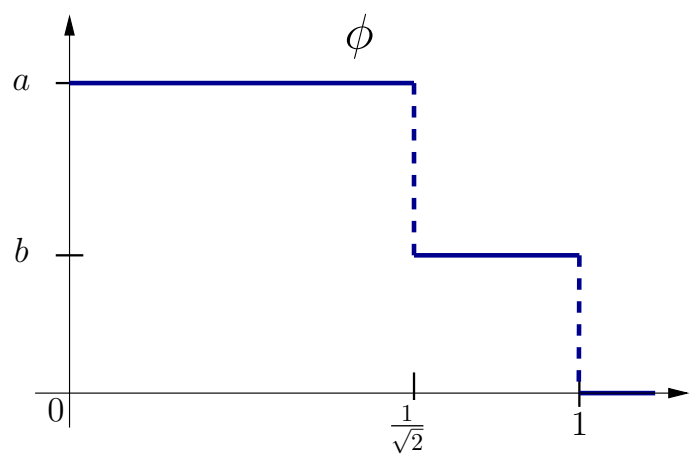

FiguRE 6.1. The worst case scenario for the decaying of the norm of the vector $\Delta$ : the formation is connected but there are two large groups with extreme values.

\section{Self-Alignment Dynamics With DiscRete time STEPS}

Models for opinion dynamics were originally introduced as a discrete algorithms. In this section we therefore extend our results on the semi-discrete continuous opinion dynamics $1.2 \mathrm{~b}$ to the fully discrete case,

$$
\frac{\mathbf{x}_{i}(t+\Delta t)-\mathbf{x}_{i}(t)}{\Delta t}=\alpha \frac{\sum_{j} \phi_{i j}\left(\mathbf{x}_{j}(t)-\mathbf{x}_{i}(t)\right)}{\sum_{j} \phi_{i j}} .
$$

In particular, for $\alpha=1 / \Delta t$ we find that $\mathbf{x}_{i}^{n}=\mathbf{x}_{i}(n \Delta t)$ satisfies the Krause model [11, 12, 75]

$$
\mathbf{x}_{i}^{n+1}=\frac{\sum_{j} \phi_{i j} \mathbf{x}_{j}^{n}}{\sum_{j} \phi_{i j}}, \quad \phi_{i j}=\phi\left(\left|\mathbf{x}_{j}^{n}-\mathbf{x}_{i}^{n}\right|\right) .
$$

In the following, we study the properties of the discrete dynamics $(7.2)$.

7.1. Consensus with global interactions. Many results of the continuous dynamics $(1.2 \mathrm{~b})$ can be translated to the discrete dynamics $(7.2)$. For example, the convex hull of the opinions $\Omega(2.3)$ is still decreasing in time:

$$
\Omega(n+1) \subset \Omega(n) .
$$

The discrete dynamics 7.2 will also converge to a consensus if initially all agents interact with each other. More precisely, arguing along the lines of proposition 2.4 gives the following result.

Theorem 7.1. Assume that $m=\min _{r \in[0,[\mathbf{x}(0)]]} \phi(r)>0$. Then, the diameter of the discrete dynamics (7.2) satisfies

$$
\left[\mathbf{x}^{n}\right] \leq(1-m)^{n}\left[\mathbf{x}^{0}\right] \stackrel{n \rightarrow \infty}{\longrightarrow} 0 .
$$

and convergence to a consensus, $\mathbf{x}_{i}^{n} \stackrel{n \rightarrow \infty}{\longrightarrow} \mathbf{x}^{\infty} \in \Omega(0)$ follows.

Proof. Using the contraction estimate 2.7 followed by the bound $\eta_{A} \geq \max _{\theta} \theta \cdot \lambda(\theta)$ yield

$$
\left[\mathbf{x}^{n+1}\right] \leq\left(1-\eta_{A}\right)\left[\mathbf{x}^{n}\right] \leq\left(1-\theta \cdot \lambda\left(\theta, t^{n}\right)\right)\left[\mathbf{x}^{n}\right], \quad a_{i j}=\frac{\phi_{i j}}{\sum_{\ell} \phi_{\ell j}} .
$$

Fix $\theta=m / N$ then $\Lambda(\theta)$ includes all agents, $\lambda\left(\theta, t^{n}\right)=N$, and we conclude

$$
\left[\mathbf{x}^{n+1}\right] \leq(1-m)\left[\mathbf{x}^{n}\right]
$$

which proves $(7.3)$. 
7.2. Clustering with local interactions. As in the continuous dynamics, we would like to investigate the behavior of the discrete dynamics (7.2) with local interactions; in particular, we are interested in the formation of clusters. Our aim is to reproduce the discrete analog of proposition 3.1 .

Proposition 7.2. Let $\mathcal{P}^{n}=\left\{\mathbf{x}_{k}^{n}\right\}_{k}$ be the solution of the discrete opinion dynamics (7.2) with compactly supported influence function $\operatorname{Supp}\{\phi(\cdot)\}=[0, R)$. Assume that it approaches a steady state fast enough so that

$$
\sum_{n=m}^{\infty} \sum_{i}\left|\mathbf{x}_{i}^{n+1}-\mathbf{x}_{i}^{n}\right| \stackrel{m \rightarrow \infty}{\longrightarrow} 0 .
$$

Then $\left\{\mathbf{x}^{n}\right\}$ approaches a stationary state, $\mathbf{x}^{\infty}$, which is partitioned into clusters, $\left\{\mathcal{C}_{k}\right\}_{k}$, such that $\{1,2, \ldots, N\}=\cup_{k=1}^{K} \mathcal{C}_{k}$ and

$$
\mathbf{x}_{i}^{n} \longrightarrow \mathbf{x}_{\mathcal{C}_{k}}^{\infty}, \quad \text { for all } i \in \mathcal{C}_{k} .
$$

Proof. By assumption 7.4

$$
\left|\mathbf{x}_{i}^{n_{2}}-\mathbf{x}_{i}^{n_{1}}\right| \leq \sum_{n=n_{1}}^{n_{2}-1}\left|\mathbf{x}_{i}^{n+1}-\mathbf{x}_{i}^{n}\right| \ll 1, \text { for } n_{2}>n_{1} \gg 1,
$$

and hence $\mathbf{x}^{n}$ approach a limit, $\mathbf{x}_{i}^{n} \stackrel{n \rightarrow \infty}{\longrightarrow} \mathbf{x}_{i}^{\infty}$. The discrete dynamics 7.2 can be written in the following form:

$$
\sum_{j} \phi_{i j}\left(\mathbf{x}_{i}^{n+1}-\mathbf{x}_{i}^{n}\right)=\sum_{j} \phi_{i j}\left(\mathbf{x}_{j}^{n}-\mathbf{x}_{i}^{n}\right) .
$$

Taking the scalar product against $\mathbf{x}_{i}^{n}$, summing in $i$ and using the symmetry of $\phi_{i j}$ yields

$$
\sum_{i j} \phi_{i j}\left\langle\left(\mathbf{x}_{i}^{n+1}-\mathbf{x}_{i}^{n}\right), \mathbf{x}_{i}^{n}\right\rangle=\sum_{i j} \phi_{i j}\left\langle\mathbf{x}_{j}^{n}-\mathbf{x}_{i}^{n}, \mathbf{x}_{i}^{n}\right\rangle=-\frac{1}{2} \sum_{i j} \phi_{i j}\left|\mathbf{x}_{j}^{n}-\mathbf{x}_{i}^{n}\right|^{2} .
$$

Since $\phi_{i j}, \mathbf{x}_{i}^{n}$ and by assumption, the tail $\sum_{m}^{\infty}\left|\mathbf{x}_{i}^{n+1}-\mathbf{x}_{i}^{n}\right|$ are bounded, we conclude that the sum on the right converges to zero

$$
\phi_{i j}\left|\mathbf{x}_{j}^{n}-\mathbf{x}_{i}^{n}\right|^{2} \stackrel{n \rightarrow \infty}{\longrightarrow} \phi\left(\mathbf{x}_{j}^{\infty}-\mathbf{x}_{i}^{\infty} \mid\right)\left|\mathbf{x}_{j}^{\infty}-\mathbf{x}_{i}^{\infty}\right|^{2}=0 .
$$

Hence, either $\mathbf{x}_{j}^{\infty}$ and $\mathbf{x}_{i}^{\infty}$ are in separate clusters, $\left|\mathbf{x}_{j}^{\infty}-\mathbf{x}_{i}^{\infty}\right|>R$ or else, they are in the limiting point of the same cluster, say $i, j \in C_{\ell}$ so that $\mathbf{x}_{j}^{\infty}=\mathbf{x}_{i}^{\infty}$.

We now turn our attention to the convergence toward consensus for the discrete dynamics (7.2). As for the continuous dynamics (1.2), there exists a Lyapunov functional energy for the dynamics under the additional assumption that the influence function $\phi$ is non-increasing. Consequently, we deduce the analog of theorem 4.2 for the discrete dynamics.

Theorem 7.3. Let $\mathcal{P}^{n}=\left\{\mathbf{x}_{k}^{n}\right\}_{k}$ be the solution of the discrete opinion dynamics $(7.2)$ with non-increasing, compactly supported influence function $\operatorname{Supp}\{\phi(\cdot)\}=[0, R)$. If $\mathcal{P}^{n}$ remains uniformly connected for any $n$, then $\mathcal{P}^{n}$ converges to a consensus.

Proof. First, we prove that the energy functional $\mathcal{E}^{n}$ is also a Lyapunov function for the discrete dynamics:

$$
\mathcal{E}^{n}:=\sum_{i j} \Phi\left(\left|\mathbf{x}_{j}^{n}-\mathbf{x}_{i}^{n}\right|\right), \quad \Phi(r)=\int_{0}^{r} s \phi(s) d s .
$$


Introducing $\varphi\left(r^{2}\right)=\Phi(r)$, we have $\varphi(r)=\int_{0}^{\sqrt{r}} s \phi(s) d s=\frac{1}{2} \int_{0}^{r} \phi(\sqrt{y}) d y$. By assumption $\phi$ is non-increasing, thus $\varphi$ is concave-down. Therefore,

$$
\begin{aligned}
\mathcal{E}^{n+1}-\mathcal{E}^{n} & =\sum_{i j} \varphi\left(\left|\mathbf{x}_{j}^{n+1}-\mathbf{x}_{i}^{n+1}\right|^{2}\right)-\varphi\left(\left|\mathbf{x}_{j}^{n}-\mathbf{x}_{i}^{n}\right|^{2}\right) \\
& \leq \frac{1}{2} \sum_{i j} \phi\left(\left|\mathbf{x}_{j}^{n}-\mathbf{x}_{i}^{n}\right|\right)\left(\left|\mathbf{x}_{j}^{n+1}-\mathbf{x}_{i}^{n+1}\right|^{2}-\left|\mathbf{x}_{j}^{n}-\mathbf{x}_{i}^{n}\right|^{2}\right) .
\end{aligned}
$$

Using $|\mathbf{a}|^{2}-|\mathbf{b}|^{2}=\langle\mathbf{a}-\mathbf{b}, \mathbf{a}+\mathbf{b}\rangle$, we deduce:

$$
\begin{aligned}
\mathcal{E}^{n+1}-\mathcal{E}^{n} & \leq \frac{1}{2} \sum_{i j} \phi_{i j}\left\langle\Delta_{t} \mathbf{x}_{j}^{n}-\Delta_{t} \mathbf{x}_{i}^{n}, \mathbf{x}_{j}^{n+1}-\mathbf{x}_{i}^{n+1}+\mathbf{x}_{j}^{n}-\mathbf{x}_{i}^{n}\right\rangle \\
& =\sum_{i j} \phi_{i j}\left\langle\Delta_{t} \mathbf{x}_{j}^{n}, \mathbf{x}_{j}^{n+1}-\mathbf{x}_{i}^{n+1}+\mathbf{x}_{j}^{n}-\mathbf{x}_{i}^{n}\right\rangle,
\end{aligned}
$$

since $\phi_{i j}=\phi_{j i}$. Writing $\mathbf{x}_{j}^{n+1}=\mathbf{x}_{j}^{n}+\Delta_{t} \mathbf{x}_{j}^{n}$, we obtain:

$$
\mathcal{E}^{n+1}-\mathcal{E}^{n} \leq \sum_{i j} \phi_{i j}\left\langle\Delta_{t} \mathbf{x}_{j}^{n}, 2\left(\mathbf{x}_{j}^{n}-\mathbf{x}_{i}^{n}\right)+\Delta_{t} \mathbf{x}_{i}^{n}-\Delta_{t} \mathbf{x}_{j}^{n}\right\rangle
$$

Combining with the equality:

$$
\sum_{j} \phi_{i j} \Delta_{t} \mathbf{x}_{i}^{n}=\sum_{j} \phi_{i j}\left(\mathbf{x}_{j}^{n}-\mathbf{x}_{i}^{n}\right)
$$

we conclude

$$
\mathcal{E}^{n+1}-\mathcal{E}^{n} \leq \sum_{i j} \phi_{i j}\left\langle\Delta_{t} \mathbf{x}_{i}^{n},-\Delta_{t} \mathbf{x}_{i}^{n}-\Delta_{t} \mathbf{x}_{j}^{n}\right\rangle=-\sum_{i j} \phi_{i j}\left|\Delta_{t} \mathbf{x}_{i}^{n}\right|^{2}
$$

where we use once again the symmetry of the coefficients $\phi_{i j}$. Thus, $\mathcal{E}^{n}$ is decaying.

Now, we would like to combine the decay of $\mathcal{E}^{n}$ and the strong connectivity of $\mathcal{P}^{n}$. Noting $\sigma_{i}=\sum_{j} \phi_{i j}$, the equality (7.7) yields:

$$
\frac{1}{2} \sum_{i, j} \phi_{i j}\left|\mathbf{x}_{j}^{n}-\mathbf{x}_{i}^{n}\right|^{2}=\sum_{i} \sigma_{i}\left\langle\mathbf{x}_{i}^{n}, \Delta_{t} \mathbf{x}_{i}^{n}\right\rangle \leq N \max _{i}\left|\mathbf{x}_{i}^{0}\right| \sqrt{\sum_{i} \sigma_{i}\left|\Delta_{t} \mathbf{x}_{i}^{n}\right|^{2}},
$$

Thus,

$$
\mathcal{E}^{n+1}-\mathcal{E}^{n} \leq-C_{0}^{2}\left(\sum_{i, j} \phi_{i j}\left|\mathbf{x}_{j}^{n}-\mathbf{x}_{i}^{n}\right|^{2}\right)^{2}, \quad C_{0}=\frac{1}{2 N \max _{i}\left|\mathbf{x}_{i}(0)\right|} .
$$

Summing in $n$, we deduce that the sum $\sum_{i, j} \phi_{i j}\left|\mathbf{x}_{j}^{n}-\mathbf{x}_{i}^{n}\right|^{2}$ becomes arbitrarily small. To conclude, we proceed as in the proof of theorem 4.2 .

7.3. Numerical simulations of discrete dynamics. In this section we illustrate the difference between the continuous opinion model $(1.2 \mathrm{~b})$ and its discrete version $(7.2)$. To this end, we run in parallel numerical simulations of the discrete and continuous model subject to the same initial conditions.

First, we run a simulation with an influence function $\phi=\chi_{[0,1]}$ (figure 7.1). Discrete and continuous dynamics are very similar, except that there are three branches in the continuous dynamics which are not present in the discrete dynamics. For this reason, at the end of the simulation, we count 4 clusters in the discrete dynamics and only 3 in the continuous version. 
Next we use the influence function (5.1) $\phi=a \chi_{[0,1 / \sqrt{2}]}+b \chi_{[1 / \sqrt{2}, 1]}$ with $b / a=10$. Here, the discrete and continuous dynamics give very different results shown in figure 7.2. As we have seen previously, the continuous dynamics converges to a distribution with uniformly spaced clusters and then reach a consensus. In contrast, the discrete dynamics does not stabilize. Order between the opinions $\left\{\mathbf{x}_{i}\right\}_{i}$ is no longer preserved, trajectories do cross. Even though the total number of clusters has been diminished with $b / a=10$ (from 4 to 3 clusters), the effect of the ratio $b / a$ on the clustering formation is less pronounced in the discrete dynamics.
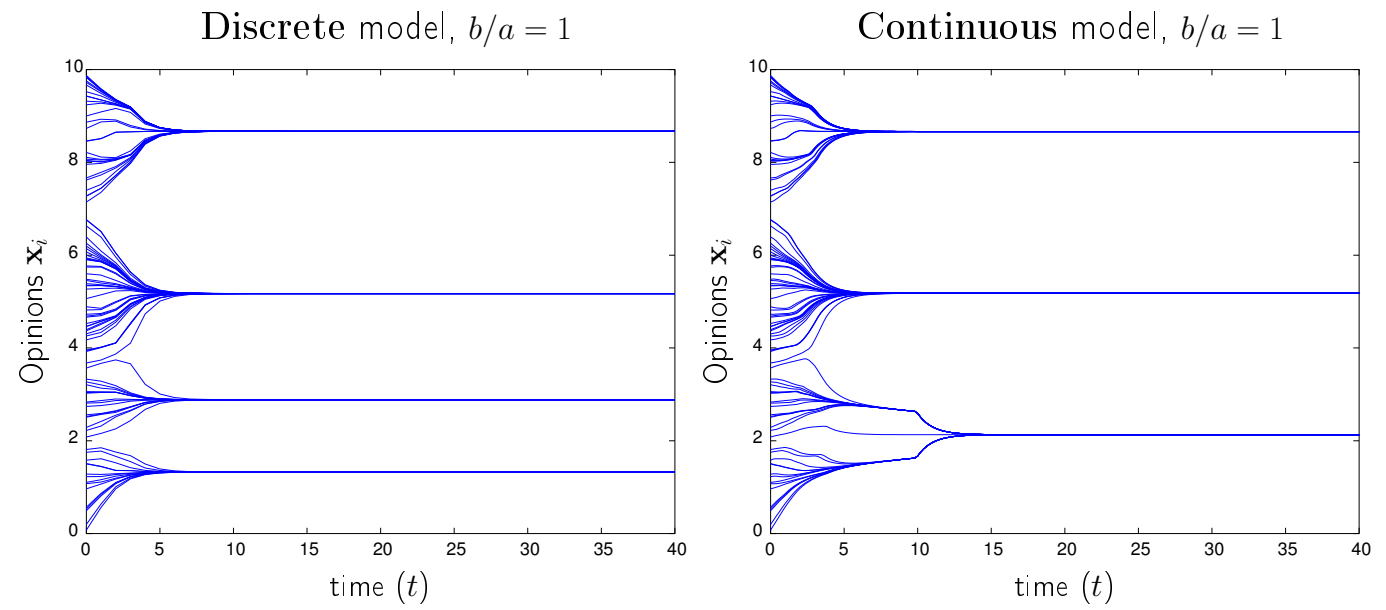

FiguRE 7.1. Simulations of the discrete (Left figure) and continuous dynamics (Right figure) with $\phi=\chi_{[0,1]}$ starting with the same initial condition. Although the two simulations are very similar, the discrete dynamics yields 4 clusters whereas the continuous dynamics gives 3 . We use a time discretization of $\Delta t=.05$ to simulate the continuous dynamics.

\section{MEAN-FIELD LIMITS: SELF-ORGANIZED HYDRODYNAMICS}

When the number of agents $N$ is large, it is convenient to describe the evolution of the resulting large dynamical systems as mean-field equation. We limit ourselves to a few classic general references on this topic [28, 57, 101], and a few recent references in the context of opinion hydrodynamics [19, 105, and in flocking hydrodynamics [22, 23, 40, 61, 73, 84, 88,

8.1. Opinion hydrodynamics. To derive the mean-field limit of the opinion dynamics model (1.2b), we introduce the so-called empirical distribution $\rho(t, \mathbf{x})$ :

$$
\rho(t, \mathbf{x}):=\frac{1}{N} \sum_{j=1}^{N} \delta_{\mathbf{x}_{j}(t)}(\mathbf{x}),
$$

where $\delta$ is a Dirac mass and $\left\{\mathbf{x}_{j}(t)\right\}_{j}$ is the solution of the consensus model $(1.2 \mathrm{~b})$. Expressed in terms of this empirical distribution, the non-symmetric model $1.2 \mathrm{~b}$ (with $\alpha=1$ ) reads,

$$
\dot{\mathbf{x}}_{i}=\frac{\int_{\mathbf{y}} \phi\left(\left|\mathbf{y}-\mathbf{x}_{i}\right|\right)\left(\mathbf{y}-\mathbf{x}_{i}\right) \rho(t, \mathbf{y}) d \mathbf{y}}{\int_{\mathbf{y}} \phi\left(\left|\mathbf{y}-\mathbf{x}_{i}\right|\right) \rho(t, \mathbf{y}) d \mathbf{y}}=\frac{(\phi(|\mathbf{y}|) \mathbf{y} * \rho)\left(\mathbf{x}_{i}\right)}{(\phi(|\mathbf{y}|) * \rho)\left(\mathbf{x}_{i}\right)} .
$$



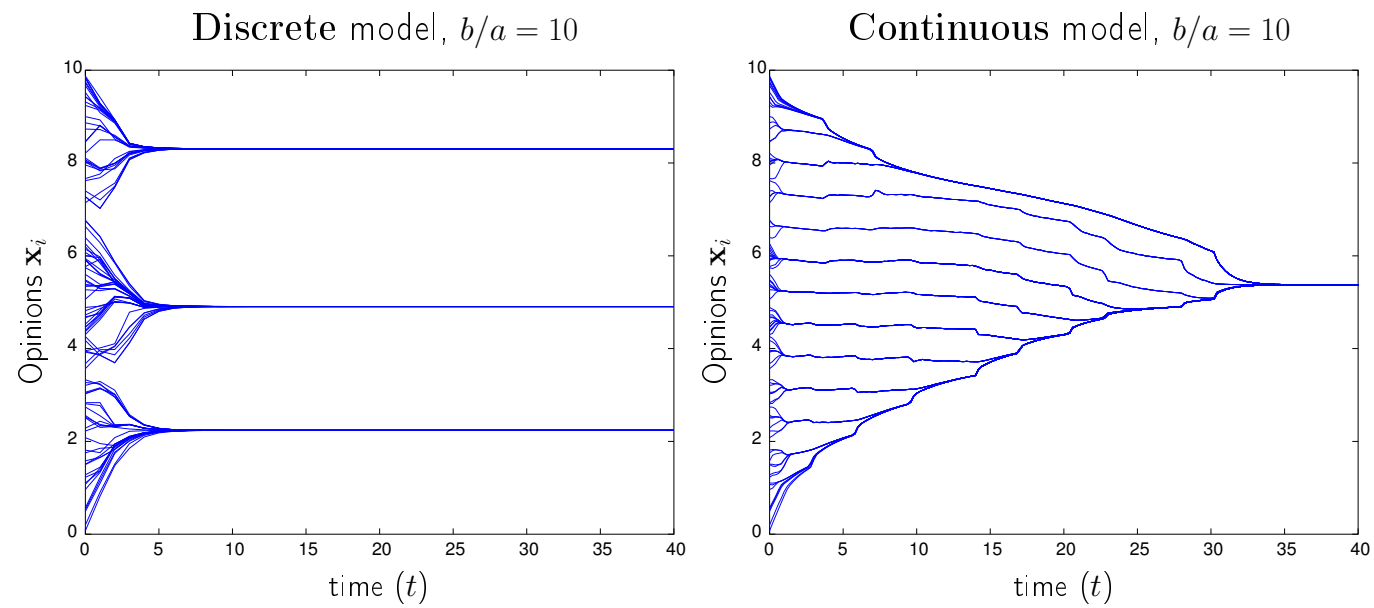

FiguRE 7.2. Simulations of the discrete (Left figure) and continuous dynamics (Right figure) with $\phi=.1 \chi_{[0,1 / \sqrt{2}]}+\chi_{[1 / \sqrt{2}, 1]}$ starting with the same initial condition. In contrast with figure 7.1, the two models produce very different output. There is no uniformly spaced formation in the discrete model, we only observe cluster formation.

This equation describes the characteristics of the density $\rho$. Indeed, integrating $\rho$ against a test function $\varphi$ yields

$$
\frac{d}{d t}(\rho, \varphi)=\frac{d}{d t}\left(\frac{1}{N} \sum_{j=1}^{N} \varphi\left(\mathbf{x}_{j}(t)\right)\right)=\frac{1}{N} \sum_{j}^{N}\left\langle\dot{\mathbf{x}}_{j}(t), \nabla_{\mathbf{x}} \varphi\left(\mathbf{x}_{j}(t)\right)\right\rangle .
$$

Using the expression (8.1), we deduce:

$$
\begin{aligned}
\frac{d}{d t}(\rho, \varphi) & =\frac{1}{N} \sum_{j=1}^{N}\left\langle\frac{\phi(|\mathbf{y}|) \mathbf{y} * \rho\left(\mathbf{x}_{j}\right)}{\phi(|\mathbf{y}|) * \rho\left(\mathbf{x}_{j}\right)}, \nabla_{\mathbf{x}} \varphi\left(\mathbf{x}_{j}(t)\right)\right\rangle=\left(\rho,\left\langle\frac{\phi(|\mathbf{y}|) \mathbf{y} * \rho}{\phi(|\mathbf{y}|) * \rho}, \nabla_{\mathbf{x}} \varphi\right\rangle\right) \\
& =\left(-\nabla_{\mathbf{x}} \cdot\left(\frac{\phi(|\mathbf{y}|) \mathbf{y} * \rho}{\phi(|\mathbf{y}|) * \rho} \rho\right), \varphi\right) .
\end{aligned}
$$

Thus, $\rho=\rho(t, \mathbf{x})$ satisfies a continuum transport equation,

$$
\partial_{t} \rho+\nabla_{\mathbf{x}} \cdot(\rho \mathbf{u})=0 \quad \text { with } \quad \mathbf{u}(\mathbf{x})=\frac{\int_{\mathbf{y}} \phi(|\mathbf{y}-\mathbf{x}|)(\mathbf{y}-\mathbf{x}) \rho(\mathbf{y}) d \mathbf{y}}{\int_{\mathbf{y}} \phi(|\mathbf{y}-\mathbf{x}|) \rho(\mathbf{y}) d \mathbf{y}} .
$$

This is the hydrodynamic description of the agent-based opinion model $(1.2 \mathrm{~b})$. Similarly, the opinion hydrodynamics of the corresponding symmetric model $1.2 \mathrm{a}$ (with $\alpha=1$ ) amounts to the aggregation model [8, 19]

$$
\partial_{t} \rho+\nabla_{\mathbf{x}} \cdot(\rho \mathbf{u})=0 \quad \text { with } \quad \mathbf{u}(\mathbf{x})=\nabla \Phi * \rho
$$

We note that the transport equations $(8.2)$ are non-linear due to the dependence of the velocity field $\mathbf{u}=\mathbf{u}(\rho)$. Main features of the particle description for opinion dynamics 1.2 carry over the hydrodynamic model $(8.2)$. Thus, for example, the symmetric model $8.2 \mathrm{~b}$ ) preserve the center of mass, $\frac{d}{d t}\left(\int_{\mathbf{x}} \mathbf{x} \rho(t, \mathbf{x}) d \mathbf{x}\right)=0$ where the non-symmetric model 8.2a does not. We

\footnotetext{
${ }^{4}(\cdot, \cdot)$ denotes the duality bracket between distributions and test functions
} 
distinguish between the two cases of global and local interactions.

The existence of regular solutions of the symmetric aggregation model $8.2 \mathrm{~b})$ for bounded decreasing $\phi$ 's such that $\left|\phi^{\prime}(r) r\right| \lesssim \phi(r)$ was proved in [8]. This holds independently whether $\phi$ is global or not. Moreover, if the kernel $\phi$ is globally supported, then one can argue along the lines of the underlying agent-based model (8.1), to prove convergence of the hydrodynamics toward a consensus, that is, $\rho(t, \mathbf{x})$ converges to a single point asymptotically in time. If $\phi$ is compactly supported, however, then the velocity field $\mathbf{u}$ need not be continuous with respect to $\rho$ due to the singularity when $\int_{\mathbf{y}} \phi(|\mathbf{y}-\mathbf{x}|) \rho(\mathbf{y}) d \mathbf{y}=0$. Then, existence and uniqueness of solution of the non-symmetric model 8.2a cannot be obtained through a standard Picard's iteration argument. The large time behavior of the dynamics in this local setup is completely open. As in the agent-based dynamics, the generic solution $\rho(t, \mathbf{x})$ is expected to concentrate in a finitely many clusters, or "islands"; in particular, under appropriate assumption on the persistence of connectivity among these islands, one may expect a consensus. Preliminary simulations show that cluster formation tends to persist for the hydrodynamic model, but analytical justification remains open.

8.2. Flocking hydrodynamics. We study the second-order flocking models $(1.3)$ in terms of the empirical distribution $f^{N}(t, \mathbf{x}, \mathbf{v}):=\frac{1}{N} \sum_{j=1}^{N} \delta_{\mathbf{x}_{j}(t)}(\mathbf{x}) \otimes \delta_{\mathbf{v}_{j}(t)}(\mathbf{v})$, where $\delta_{\mathbf{x}} \otimes \delta_{\mathbf{v}}$ is the usual Dirac mass on the phase space $\mathbb{R}^{d} \times \mathbb{R}^{d}$. Consider the non-symmetric particle model system for flocking (1.3b): expressed in terms of $f^{N}$, it reads

$$
\frac{d \mathbf{x}_{i}}{d t}=\mathbf{v}_{i}, \quad \frac{d \mathbf{v}_{i}}{d t}=\alpha F\left[f^{N}\right]\left(\mathbf{x}_{i}, \mathbf{v}_{i}\right), \quad F[f](\mathbf{x}, \mathbf{v}):=\alpha \frac{\int_{\mathbf{y}, \mathbf{w}} \phi(|\mathbf{y}-\mathbf{x}|)\left(\mathbf{w}-\mathbf{v}_{i}\right) f(\mathbf{y}, \mathbf{w}) d \mathbf{y} d \mathbf{w}}{\int_{\mathbf{y}} \phi(|\mathbf{y}-\mathbf{x}|) f(\mathbf{y}, \mathbf{w}) d \mathbf{y} d \mathbf{w}},
$$

which leads to Liouville's equation,

$$
\partial_{t} f+\mathbf{v} \cdot \nabla_{\mathbf{x}} f+\nabla_{\mathbf{v}} \cdot(F[f] f)=0 .
$$

Integrating the empirical distribution $f^{N}$ in the velocity variable $\mathbf{v}$ yields the hydrodynamic description of flocking, expressed in terms of the density and momentum distributions of particles,

$$
\begin{aligned}
\rho(t, \mathbf{x})=\int_{\mathbf{v}} f(t, \mathbf{x}, \mathbf{v}) d \mathbf{v} & \left(\text { corresponding to } \frac{1}{N} \sum_{j=1}^{N} \delta_{\mathbf{x}_{j}(t)}(\mathbf{x})\right), \\
\rho(t, \mathbf{x}) \mathbf{u}(t, \mathbf{x})=\int_{\mathbf{v}} \mathbf{v} f(t, \mathbf{x}, \mathbf{v}) d \mathbf{v} & \left(\text { corresponding to } \frac{1}{N} \sum_{j=1}^{N} \mathbf{v}_{j}(t) \delta_{\mathbf{x}_{j}(t)}(\mathbf{x})\right) .
\end{aligned}
$$

Integrating the kinetic equation (8.3) against the first moments $(1, \mathbf{v})$ yields the system, cf., 61, 23, 88,

$$
\begin{aligned}
& \partial_{t} \rho+\nabla_{\mathbf{x}} \cdot(\rho \mathbf{u})=0 \\
& \partial_{t}(\rho \mathbf{u})+\nabla_{\mathbf{x}} \cdot(\rho \mathbf{u} \otimes \mathbf{u}+\mathbf{P})=\alpha \rho(\overline{\mathbf{u}}-\mathbf{u}) .
\end{aligned}
$$

The expression on the right reflects alignment: the tendency of agents with velocity $\mathbf{u}$ to relax towards the local average velocity, $\overline{\mathbf{u}}(\mathbf{x})$, dictated by the normalized influence function $a(\mathbf{x}, \mathbf{y})$,

$$
\overline{\mathbf{u}}(\mathbf{x}):=\int_{\mathbf{y}} a(\mathbf{x}, \mathbf{y}) \rho(\mathbf{y}) \mathbf{u}(\mathbf{y}) d \mathbf{y}, \quad \int_{\mathbf{y}} a(\mathbf{x}, \mathbf{y}) \rho(\mathbf{y}) d \mathbf{y}=1 .
$$


This includes in particular, the hydrodynamic description of the symmetric and non-symmetric flocking models, given respectively by

$$
a(\mathbf{x}, \mathbf{y})= \begin{cases}\phi(|\mathbf{y}-\mathbf{x}|) & \text { C-S model 1.3a), } \\ \frac{\phi(|\mathbf{y}-\mathbf{x}|)}{\int_{\mathbf{y}} \phi(|\mathbf{y}-\mathbf{x}|) \rho(\mathbf{y}) d \mathbf{y}} & \text { non-symmetric model } 1.3 \mathrm{~b} .\end{cases}
$$

The system 8.4 is not closed since the equation for $\rho \mathbf{u} 8.4 \mathrm{~b}$ does depend on the third moment of $f$ which is encoded in the pressure term $\mathbf{P}:=\int_{\mathbf{v}}(\mathbf{v}-\mathbf{u}) \otimes(\mathbf{v}-\mathbf{u}) f(t, \mathbf{x}, \mathbf{v}) d \mathbf{v}$. If we neglect the pressure (in other words, assume a monophase distribution, $f(t, \mathbf{x}, \mathbf{v})=$ $\rho(t, \mathbf{x}) \delta_{\mathbf{u}(t, \mathbf{x})}(\mathbf{v})$ so that $\left.\mathbf{P} \equiv 0\right)$, then the flocking hydrodynamics $(8.4)$ is reduced to the closed system

$$
\left\{\begin{array}{l}
\partial_{t} \rho+\nabla_{\mathbf{x}} \cdot(\rho \mathbf{u})=0, \\
\partial_{t} \mathbf{u}+\left(\mathbf{u} \cdot \nabla_{\mathbf{x}}\right) \mathbf{u}=\alpha(\overline{\mathbf{u}}-\mathbf{u}) .
\end{array}\right.
$$

The question of an emerging flock in (8.5) follows along the lines of our discussion on the underlying agent-based models (1.3). The case of a global influence function is rather wellunderstood: in particular, regularity of the one-dimensional "incompressible" case, $\rho \equiv 1$, depends on initial critical threshold [81, 96. Flocking hydrodynamics governed by locally supported influence function requires a more intricate analysis, due to the realistic presence of vacuum, [102]. The hydrodynamic description of self-organized dynamics give rise to systems like (8.5) which involve nonlocal means. Questions of regularity and quantitative behavior of such systems provide a rich source for future studies.

\section{FurThER READING ON SELF-ORGANIZED DYNAMICS}

In this paper we discussed fundamental aspects which arise in the context of flocking and opinion dynamics, as prototype models for self-organized dynamics. Specifically, we focused here on the emerging large-time behavior of self-alignment and we highlight a few open questions aiming to attract further mathematical studies in this direction. The much broader subject of self-organized dynamics lies at the crossroads of several fields. A comprehensive review of the subject is beyond the scope of this paper, in particular, as it continues to attract an increasing amount of attention reported in a rapidly growing literature. Instead, we refer the interested reader to a selection of references outlined below. As with all multidisciplinary fields, the work on self-organized dynamics can be classified into several different categories. We shall mention five of them.

Different disciplines. A natural classification is offered by the underlying topic. Many models of self-organized dynamics are driven by examples from biology: these include aggregation of bacteria and amoeba [6, 51, 59, 74, 98, dynamics of insects [14, 34, school of fish [1, 67, 111] flocking of birds [4, 25, 26, 27, 37, 38, 61, 93, 103, 107], and related models in ecology [58]. Self-organized dynamics found its in many other areas, from pedestrian and traffic dynamics [64, 92, social networks and economics [48, 66, 69, 77, 83, complex networks [5, 44, 90] and opinion dynamics [7, 24, 42, 49, 50, 63, 75, 105, 109, 110], all the way to applications in marketing [2, 3, production networks [94, robotics [33, 71, 113] and materials [99, 85], and with somewhat more esoteric examples such as gossiping [13, collective motion at heavy metal concerts [100] and self-organized phases in the Tour De France [106]. 
Different models. Together with the different contexts, come different models of selforganized dynamics. We mention a few of the more notable ones: Krause model for opinion dynamics [75] and the follow-up works in [12, 19, 63, 76, 82, Axelrod models for marketing [2] and the influential models for "flocking" (at various "levels") of Aoki, Reynolds and Couzin [1, 34, 36, 80, 93, 111, Vicsek et. al, [107] and the follow-up works in [40, 41, 70], Cucker-Smale model [37, 38] and related works in [10, 22, 60, 61, 62, 73, 88, 97, and the StarFlag project [4, 25, 26, 27].

Different scales. Different models of self-organized dynamics are realized at different scales. As examples for agent-based models (also known as Individual-Based Models (IBM)) we mention [7, 34, 58, 75, 79, 91, 93. Their mean-field limit leads to a kinetic description [21, 22, 49, 61, 105] and macroscopic averaging then leads to hydrodynamic-scale description as in [15, 23, 40, 41, 51, 73, 74, 78, 84, 85, 102.

Different approaches. In this paper, we focused our attention on mathematical aspects which explain the large time behavior of self-alignment models. The study of general models for self-organized dynamics includes several different approaches. Classified by the tools of the trade, we mention statistical mechanics [10, 24, 101, clustering and spectral theory of graphs [15, 32, 33, 70, 91, optimization and control [17, 43, 44, 71, 72, 90, 113], game theory [5, 65], jump processes, nonlinear Markov chains and stochastic analysis [15, 52, 66, 107.

Different patterns. One of the most intriguing features of self-organized dynamics is the formation of different patterns. In this paper, we limited ourselves to the simple pattern of "consensus" (or a "flock") but the format is much richer. We mention the example of swarming and mill-like vortices [18, 21, 23, 47, 50, 78, 79, 80, 98, 104], phase transition [55, 107, aggregation [15], biotic colonies [6, 74], lattices [89], leaders [35, 97], shocks [9, 102] and related issues which arise in the context of control and stability [11, 47, 72, 79].

Finally, we recommend on several reviews on self-organization [16, 24, 151, 66, 110], and in particular, the most recent comprehensive review of Vicsek and Zefeiris [108].

\section{REFERENCES}

[1] I. Aoki, A simulation study on the schooling mechanism in fish Bull. Japanese Society of Scientific Fisheries, 48(8), 1081-1088, 1982.

[2] R. Axelrod The Evolution of Cooperation, New York: Basic Books.

[3] R. Axelrod The Complexity of Cooperation: Agent-based models of competition and collaboration, Princeton University Press, Princeton, NJ.

[4] M. Ballerini, N. Cabibbo, R. Candelier, A. Cavagna, E. Cisbani, I. Giardina, V. Lecomte, A. Orlandi, G. Parisi, A. Procaccini, M. Viale and V. Zdravkovic, Interaction ruling animal collective behavior depends on topological rather than metric distance PNAS, 105(4), 1232-1237, 2008.

[5] N. Bellomo, M. Herrero and A. Tosin On the dynamics of social conflicts: looking for the black swan, Kinetic And Related Models 6:459-479, 2013.

[6] E. Ben-Jacob Bacterial self-organization: co-enhancement of complexification and adaptability in a dynamic environment. Phil. Trans. R. Soc. Lond. A., 361(1807):1283-1312, 2003.

[7] E. Ben-Naim, Opinion dynamics: rise and fall of political parties Europhys. Lett., 69(5):671-677, 2005.

[8] A. Bertozzi, J. Carrillo and T. Laurent, Blow-up in multidimensional aggregation equations with mildly singular interaction kernels Nonlinearity 22 (2009) 683-710.

[9] A. Bertozzi, J. Rosado, M. Short and L. Wang, Contagion shocks in one dimension preprint

[10] W. Bialek, A. Cavagna, I. Giardina, T. Mora, O. Pohl, E. Silvestri, M. Viale, A. Walczak, Social interactions dominate speed control in driving natural flocks toward criticality ArXiv:1307.5563v1.

[11] V. Blondel, J. M Hendricks, A. Olshevsky, and J. Tsitsiklis, Convergence in multiagent coordination, consensus, and flocking. In IEEE Conference on Decision and Control, volume 44, page 2996, 2005.

[12] V. D. Blondel, J. M. Hendricks, and J. N. Tsitsiklis, On Krause's multi-agent consensus model with statedependent connectivity. Automatic Control, IEEE Transactions on, 54(11):2586-2597, 2009. 
[13] S. Boyd, A. Ghosh, B. Prabhakar, and D.Shah, Randomized gossip algorithms. IEEE Trans. Inform. Theory, 52;2508-2530, 2006

[14] A. M. Bruckstein, N. Cohen, A. Efrat, Ants, crickets and frogs in cyclic Pursuit CIS report \#9105, Center for Intelligent Systems, Technion Israel Inst. of Tech., 1991.

[15] M. Burger, J. Haskovec, M.-T. Wolfram, Individual based and mean-field modelling of direct aggregation, Phys. D, 260: 145-158, 2013.

[16] S. Camazine, J. L. Deneubourg, N. R Franks, J. Sneyd, G. Theraulaz, and E. Bonabeau, Self-organization in biological systems. Princeton University Press; Princeton, NJ: 2001, 2001.

[17] G. de Campos and A. Seuret Improved Consensus Algorithms using Memory Effects, Decision and Control 2011 50th IEEE Conf. (CDC-ECC), IEEE, 982-987, 2011

[18] J. A. Canizo, J. A. Carrillo and J. Rosado, Collective behavior of animals: swarming and complex patterns. 2009.

[19] C. Canuto, F. Fagnani, and P. Tilli, An Eulerian approach to the analysis of Krause's consensus models SIAM J. Control Optim., 50(1), 243-265. 2012.

[20] R. Carli, F. Fagnani, A. Speranzon, and S. Zampieri, Communication constraints in the average consensus problem. Automatica, 44, 671-684, 2008.

[21] J. A. Carrillo, M. D'Orsogna, V. Panferov, Double milling in self-propelled swarms from kinetic theory. Kinet Relat Models, 2:363-378, 2009.

[22] J. A. Carrillo, M. Fornasier, J. Rosado, and G. Toscani, Asymptotic flocking dynamics for the kinetic Cucker-Smale model. SIAM J. Math. Anal., 42:218-236, 2010.

[23] J. A. Carrillo, M. Fornasier, G. Toscani, and F. Vecil, Particle, kinetic, and hydrodynamic models of swarming. in Naldi, G., Pareschi, L., Toscani, G. (eds.) Mathematical Modeling of Collective Behavior in Socio-Economic and Life Sciences, Series: Modelling and Simulation in Science and Technology, Birkhauser, (2010), 297-336.

[24] C. Castellano, S. Fortunato, V. Loreto, Statistical physics of social dynamics, Rev. Modern Phys, 81:591646, 2009.

[25] A. Cavagna, I. Giardina, A. Orlandi, G. Parisi, A. Procac-cini, M. Viale and V. Zdravkovic, The starflag handbook on collective animal behaviour. 1: Empirical methods. Animal Behaviour 76:217-236, 2008.

[26] A. Cavagna, I. Giardina, A. Orlandi, G. Parisi and A. Procaccini, The starflag handbook on collective animal behaviour. 2: Three-dimensional analysis. Animal Behaviour 76:237-248, 2008.

[27] A. Cavagna, A. Cimarelli, I. Giardina, G. Parisi, R. Santagati, F. Stefanini and M. Viale, Scale-free correlations in starling flocks. Proc. Nat. Academy Sci., U.S.A. 107:11865-11870, 2010.

[28] C. Cercignani, R. Illner, M. Pulvirenti, The mathematical theory of dilute gases. Springer series in Applied Mathematical Sciences, 106, Springer-Verlag, 1994.

[29] Fan R. K. Chung, Spectral Graph Theory Amer. Math. Soc. CBMS Regional Conference Series in Mathematics, No. 92), 1997

[30] J.E. Cohen, Y. Derriennic and Gh. Zbaganu, Majorization, Monotonicity of Relative Entropy, and Stochastic Matrices Contemp. Mathematics 149, 251-259, 1993.

[31] J. E. Cohen, Y. Iwasa, Gh. Rautu, M. B. Ruskai, E. Seneta and Gh. Zbaganu, Relative entropy under mappings by stochastic matrices Linear Algebra Applications, 179(15) 211-235, 1993.

[32] D. Comaniciu and P. Meer, Mean shift: A robust approach toward feature space analysis. IEEE Transactions on Pattern Analysis and Machine Intelligence archive Volume 24(5) Pages 603 - 619, 2002.

[33] J. Cortés, S. Martinez, and F. Bullo, Robust rendezvous for mobile autonomous agents via proximity graphs in arbitrary dimensions, IEEE Trans. Automat. Control, 51, 1289-1298, 2006.

[34] I. Couzin and N. Franks Self-organized lane formation and optimized traffic flow in army ants Proc. $R$. Soc. Lond. B, 270:139-146, 2003.

[35] I.D. Couzin, J. Krause, N.R. Franks, and S.A. Levin, Effective leadership and decision-making in animal groups on the move. Nature, 433(7025):513-516, 2005.

[36] I. D. Couzin, J. Krause, R. James, G. D. Ruxton and N. R. Franks, Collective memory and spatial sorting in animal groups J. of Theoretical Biology, 218(1), 1-11, 2002.

[37] F. Cucker and S. Smale. Emergent behavior in flocks. IEEE Transactions on automatic control, 52(5):852, 2007.

[38] F. Cucker and S. Smale, On the mathematics of emergence. Japanese Journal of Mathematics, 2(1):197-227, 2007.

[39] F. Cucker, S. Smale and D.X. Zhou, Modeling language evolution. Found. Comput. Math., 4, 315-343, 2006. 
[40] P. Degond and S. Motsch, Continuum limit of self-driven particles with orientation interaction Math. Models Methods Appl. Sci., 18(1):1193-1215, 2008.

[41] P. Degond and S. Motsch, A macroscopic model for a system of swarming agents using curvature control J. Stat. Physics, 143(4):685-714,2011.

[42] M. H. DeGroot, Reaching a consensus J. Amer. Stat. Association, 69(345): 118-121, 1974.

[43] P. DeLellis, M. diBernardo and F. Garofalo Novel decentralized adaptive strategies for the synchronization of complex networks Automatica 45(5):1312-1318, 2009.

[44] P. DeLellis, M. diBernardo, F. Garofalo, D. Liuzza Analysis and stability of consensus in networked control systems Applied Mathematics and Computation 217(3):988-1000, 2010.

[45] J. Demmel. Applications of Parallel Computers. Lecture notes http://www.cs.berkeley.edu/ demmel/cs267/lecture20/lecture20.html.

[46] R.L. Dobrushin Central limit theorem for nonstationary Markov chains.I Theory Probab. Appl., 1(1), 65-80, 1956.

[47] D’Orsogna, M. R., Chuang, Y.-L., Bertozzi, A. L. and Chayes, L., Self-propelled particles with soft-core interactions. patterns, stability, and collapse. Phys. Rev. Lett. 96:104-302, 2006.

[48] A. A. Dragulescu and V. M. Yakovenko Statistical mechanics of money The European Physical Journal B, 17:723-729, 2000.

[49] B. Duering, P. Markowich, J.F. Pietschmann, and M.T. Wolfram, Boltzmann and Fokker-Planck equations modelling opinion formation in the presence of strong leaders. Proceedings of the Royal Society A: Mathematical, Physical and Engineering Science, 465(2112):3687, 2009.

[50] L. Edelstein-Keshet, Mathematical models of swarming and social aggregation, International Symposium on Nonlinear Theory and its Applications, (NOLTA 2001) Miyagi, Japan, 2001.

[51] R. Eftimie, Hyperbolic and kinetic models for self-organized biological aggregations and movement: a brief review. J Math Biol. 65(1):35-75, 2012.

[52] F. Fagnani and S. Zampieri, Randomized consensus algorithms over large scale networks. IEEE J. Selected Areas of Communications, 26, 634-649, 2008.

[53] M. Fiedler, Algebraic connectivity of graphs, Czech. Math. J. 23(98), 1973, pp. 298-305.

[54] M. Fiedler, Laplacian of graphs and algebraic connectivity. Combinatorics and Graph Theory 25, 57-70, 1989.

[55] A. Frouvelle and J.-G. Liu Dynamics in a kinetic model of oriented particles with phase transition, SIAM J. Math Anal., 44:791-826, 2012.

[56] C. Godsil and G. Royle, Algebraic Graph Theory Graduate Texts in Mathematics ser. vol. 207. .SpringerVerlag, 2001.

[57] F. Golse, The mean-field limit for the dynamics of large particle systems. Journées Équations aux dérivés partielles, 9:1-47, 2003.

[58] V. Grimm and S. F Railsback, Individual-based modeling and ecology. Princeton Univ Pr, 2005.

[59] S. Y Ha and D. Levy Particle, kinetic and fluid models for phototaxis, Discrete Cont. Dynamical Systems Ser. B., 12(1):77-108, 2009

[60] S. Y Ha and J. G Liu, A simple proof of the Cucker-Smale flocking dynamics and mean-field limit. Communications in Mathematical Sciences, 7(2):297-325, 2009.

[61] S. Y. Ha and E. Tadmor, From particle to kinetic and hydrodynamic descriptions of flocking. Kinetic and Related Models, 1(3):415-435, 2008.

[62] J. Haskovec Flocking dynamics and mean field limit of the Cucker-Smale-type model with topological interactions, Phys. D, 261: 42-51, 2013.

[63] R. Hegselmann and U. Krause, Opinion dynamics and bounded confidence: models, analysis and simulation. Journal of Artificial Societies and Social Simulation, 5(3), 2002.

[64] D. Helbing, Traffic and related self-driven many particle systems. Reviews of Modern Physics, 73, 1067-1141, 2001.

[65] D. Helbing, Pattern formation, social forces, and diffusion instability in games with success-driven motion, Eur. Phys. J. B, 67:345-356, 2009

[66] D. Helbing Quantitative Sociodynamics: Stochastic Methods and Models of Social Interaction Processes Springer-Verlag,2010.

[67] C. K Hemelrijk and H. Hildenbrandt, Self-organized shape and frontal density of fish schools. Ethology, 114(3):245-254, 2008. 
[68] J. H. Hubbard and B. H. West, Differential Equations: A Dynamical Systems Approach. Springer Verlag, 1997.

[69] M. O. Jackson, Social and Economic Networks. Princeton University Press, 2010.

[70] A. Jadbabaie, J. Lin, and A.S. Morse, Coordination of groups of mobile autonomous agents using nearest neighbor rules. IEEE Trans. Automat. Control, 48, 988-1001, 2003.

[71] M. Ji and M. Egerstedt, Distributed coordination control of multi-agent systems while preserving connectedness, IEEE Trans. Robot., vol. 23(4), 693-703, 2007.

[72] E.W. Justh and P.S. Krishnaprasad Extremal Collective Behavior, in Proc. 49th IEEE Conf. Decision and Control, 5432-5437, 2010.

[73] T. Karper, A. Mellet and K. Trivisa, Hydrodynamic limit of the kinetic Cucker-Smale flocking model ArXiv:1205.6831, 2012.

[74] D. A. Kessler and H. Levine, Pattern Formation in Dictyostelium via the Dynamics of Cooperative Biological Entities, Phys. Rev. E 48(6):4801-4804, 1993.

[75] U. Krause, A discrete nonlinear and non-autonomous model of consensus formation. Communications in difference equations, page 227-236, 2000.

[76] S. Kurz and J. Rambau On the Hegselmann-Krause conjecture in opinion dynamics J. Difference eqs. Appl, 17(6):859-876, 2011.

[77] P. F. Lazarsfeld, R. K. Merton, Friendship as a social process: a substantive and methodological analysis. In "Freedom and Control in Modern Society" (M. Berger, T. Abel, and C. H. Page, eds.) New York, Van Nostrand, 18-66, 1954.

[78] H. Levine, W.-J. Rappel, I. Cohen, Self-organization in systems of self-propelled particles Phys. Rev. E 63:017101, 2000.

[79] W. Li, Stability analysis of swarms with general topology IEEE Trans. Systems, Man, Cyber., Part B, $38(4), 2008$.

[80] X. Li and J. Xiao, Swarming in homogeneous environments: A social interaction based framework Journal Theor. Biology, 264(3):747-759, 2010.

[81] H. Liu and E. Tadmor, Critical thresholds in convolution model for nonlinear conservation laws, SIAM J. Math. Anal. 33(4), 930-945, 2001.

[82] J.Lorenz, Continuous opinion dynamics of multidimensional allocation problems under bounded confidence. A survey. Internat. J. Modern Phys. C, 18, 1819-1838, 2007.

[83] M. McPherson, L. Smith-Lovin, and J. M Cook, Birds of a Feather: Homophily in Social Networks. Annual Review of Sociology, Vol. 27: 415-444 2001.

[84] N. Mecholsky, E. Ott and T. M. Antonsen, Obstacle and predator avoidance in a model for flocking Physica D, 239, 988-996, 2010.

[85] T. Mengesha and Q. Du, Analysis of a scalar peridynamic model with a sign changing kernel preprint

[86] R. Merris Laplacian Matrices of Graphs: A Survey Linear Algebra Applications 197,198, 143-176, 1994.

[87] B. Mohar, Eigenvalues, diameter, and mean distance in graphs, Graph and Combinatorics 7:53-64, 1991.

[88] S. Motsch and E. Tadmor, A new model for self-organized dynamics and its flocking behavior. Journal of Statistical Physics, 144(5):923-947, August 2011.

[89] R. Olfati-Saber, Flocking for multi-agent dynamic systems: algorithms and theory. IEEE Trans. Auto. Control 51(3), 401-420, 2006.

[90] R. Olfati-Saber and R.M. Murray Consensus problems in network of agents with switching topology and time delays. IEEE Trans. on Automatic Control 49(9):1520-1533, 2004.

[91] R. Olfati-Saber, J.A. Fax, and R.M. Murray, Consensus and cooperation in networked multi-agent systems. Proc. IEEE, 95, 215-233, 2007.

[92] B. Piccoli and A. Tosin, Time-evolving measures and macroscopic modeling of pedestrian flow. Arch. Rat. Mech. Anal., 199, 707-738, 2011

[93] C. W. Reynolds, Flocks, herds and schools: A distributed behavioral model. In ACM SIGGRAPH Computer Graphics, 21, 25-34, 1987.

[94] C. Ringhofer Traffic flow models and service rules for complex production systems, Decision Policies for Production Networks, (K. Kempf, D. Armbruster eds), pp.209-233, Springer 2012

[95] S. E. Schaeffer Survey graph clustering Computer Science Review 1, 27-64, 2007.

[96] S. Schochet and E. Tadmor, The regularized Chapman-Enskog expansion for scalar conservation laws, Arch. Rational Mech. Anal. 119, 95-107, 1992.

[97] J. Shen Cucker-Smale flocking under hierarchical leadership SIAM J. Appl. Math., 68(3):694-719, 2007. 
[98] A. Shklarsh, G. Ariel,, E. Schneidman and E. Ben-Jacob Smart swarms of bacteria-inspired agents with performance adaptable interactions, PLoS Computational Biology 7(9):1-11, 2011.

[99] S. A. Silling, Reformulation of Elasticity Theory for Discontinuities and Long-Range Forces. J. Mech. Physics of Solids, 48, 175-209, 2000.

[100] J. L. Silverberg, M. Bierbaum, J. Sethna, and I. Cohen, Collective motion of humans in mosh and circle pits at heavy metal concerts, Phys. Rev. Lett., 110: 228701, 2013.

[101] H. Spohn, Large Scale Dynamics of Interacting Particles, Texts and Monographs in Physics, Springer 1991.

[102] E. Tadmor and C. Tan, Critical thresholds in flocking hydrodynamics with nonlocal alignment, ArXiv1403.0991v1.

[103] J. Toner and Y. Tu, Flocks, herds, and schools. A quantitative theory of flocking, Physical Review E. 58, 4828-4858 (1998).

[104] C. M. Topaz and A. L. Bertozzi, Swarming patterns in a two-dimensional kinematic model for biological groups. SIAM J. Appl. Math. 65, 152-174 (2004).

[105] G. Toscani, Kinetic models of opinion formation. Comm. Math. Sci., 4,481-496, 2006.

[106] H. Trenchard Peloton phase oscillations Chaos,Solitons \& Fractals, 56: 194-201, 2013.

[107] T. Vicsek, A. Czirók, E. Ben-Jacob, I. Cohen, and O. Shochet, Novel type of phase transition in a system of self-driven particles, Physical Review Letters, 75(6), 1226-1229, 1995.

[108] T. Vicsek and A. Zefeiris, Collective motion. Physics Reprints, 517:71-140(2012).

[109] G. Weisbuch, G. Deffuant and F. Amblard, Persuasion dynamics Phys. A, 353:555-575, 2005.

[110] G. Weisbuch Social opinion dynamics in "Econophysics and Sociophysics: Trends and Perspectives" (B. K. Chakrabarti, A. Chakrabarti and A. Chatterjee, eds), Wiley, 2006, 67-94.

[111] L. Youseff, A. Barbaro, P. Trethewey, B. Birnir, and J. Gilbet, Parallel modeling of fish interaction, Computational Science and Engineering, 11th IEEE International Conference, 234-241, 2008.

[112] M. Zavlanos G.Pappas Potential Fields for Maintaining Connectivity of Mobile Networks. EEE Transactions on Robotics 23(4), 812-816, 2007.

[113] M. Zavlanos, M. Egerstedt, and G. J. Pappas, Graph theoretic connectivity control of mobile robot networks. Proceedings of the IEEE, 99(9):1525-1540, 2011.

(Sebastien Motsch)

School of Mathematical \& Statistical Sciences

Arizona State University

TEMPE, AZ 85287 USA

E-mail address: Sebastien.Motsch@asu.edu

$U R L:$ http://www.seb-motsch.com

(Eitan Tadmor)

Center for Scientific Computation And Mathematical Modeling (CSCAMM)

AND

Department of Mathematics, Institute for Physical Science and Technology

University of Maryland, College Park, MD 20742 USA

E-mail address: tadmor@cscamm.umd.edu

$U R L$ : http://www. cscamm.umd.edu/tadmor 